\title{
Numerical Analysis of Nonlinear Multiharmonic Eddy Current Problems*
}

\author{
F. Bachinger \\ U. Langer \\ J. Schöberl
}

April 2004

\begin{abstract}
This work provides a complete analysis of eddy current problems, ranging from a proof of unique solvability to the analysis of a multiharmonic discretization technique.

For proving existence and uniqueness, we use a Schur complement approach in order to combine the structurally different results for conducting and non-conducting regions.

For solving the time-dependent problem, we take advantage of the periodicity of the solution. Since the sources usually are alternating current, we propose a truncated Fourier series expansion, i.e. a so-called multiharmonic ansatz, instead of a costly time-stepping scheme. Moreover, we suggest to introduce a regularization parameter for the numerical solution, what ensures unique solvability not only in the factor space of divergence-free functions, but in the whole space $\boldsymbol{H}$ (curl). Finally, we provide estimates for the errors that are due to the truncated Fourier series, the spatial discretization and the regularization parameter.
\end{abstract}

\section{Introduction}

This paper aims at providing a complete analysis of general nonlinear eddy current problems and their numerical treatment by a multiharmonic solution technique. It summarizes and extends the results that were obtained in [3]. Corresponding to the following main tasks, the paper splits into four parts:

- Analysis of existence and uniqueness,

- Time discretization by means of a truncated Fourier series expansion,

- Regularization and complete analysis of the linear problem,

- A combined error estimate with respect to the truncation, spatial discretization regularization parameter.

*This work has been supported by the Austrian Science Fund "Fonds zur Förderung der wissenschaftlichen Forschung (FWF)" under the grants SFB F013, P 14953 and START Y192. 
Concerning the first item, we point out that eddy current problems are of parabolic type in conducting regions and reduce to an elliptic problem in nonconducting domains. Although PDEs of mixed type have been widely analyzed (e.g. [17]), there has not been published a simple proof of unique solvability of general eddy current problems yet, at least to our knowledge. In Section 2, we rigorously analyze the problem and present a new proof of existence and uniqueness in domains consisting of both conducting and non-conducting parts.

It is worth mentioning that in many real life problems, the sources are harmonic alternate current. Consequently, the solution would be harmonic as well, provided the problem was linear. Due to the generally nonlinear relation between magnetic field and induction, however, the solution is not necessarily harmonic, but can be represented as a Fourier series.

This gives rise to the idea of a so-called multiharmonic ansatz, i.e. of a truncation of the Fourier series, instead of an expensive time-stepping scheme. Since only a fairly small number of harmonics is required for sufficiently good approximation, this approach easily outperforms time-stepping schemes.

The simulation of electromagnetic devices in the frequency domain, i.e. by means of a harmonic or multiharmonic ansatz, has been pursued e.g. by Yamada and Bessho in [25] or Gyselinck et al. in [14]. Other works on this topic include for example $[4,11,13,18,24]$. Whereas most of these works consider the problem in complex vector spaces, we propose a real scheme because of the easier linearization: Since the operators do not satisfy the Cauchy-Riemann equations, the complex problem is not differentiable and thus cannot be linearized by the Newton method (cf. e.g. $[13,16])$.

We present and analyze the multiharmonic ansatz in Section 3, where we also provide an estimate of the error due to truncation of the Fourier series.

As far as regularization is concerned, we remark that the problem is uniquely solvable only in the factor space of divergence-free functions. As will be seen in Section 2, this is due to the kernel of the curl-operator. In order to avoid working in this factor space, we regularize the problem, what ensures unique solvability in the whole space $\boldsymbol{H}$ (curl).

In Section 4, we justify this procedure for the example of the linear harmonic problem and present estimates of the additional error that is introduced by this regularization.

Finally, in Section 5, we combine the results on the truncated Fourier series, on regularization and on finite element discretization in order to provide a complete error estimate. 


\section{Existence and Uniqueness}

Eddy current problems are described by the quasistationary Maxwell equations (e.g. [15])

$$
\begin{aligned}
\operatorname{curl} \boldsymbol{H} & =\boldsymbol{J}, \\
\operatorname{curl} \boldsymbol{E} & =-\frac{\partial \boldsymbol{B}}{\partial t}, \\
\operatorname{div} \boldsymbol{B} & =0,
\end{aligned}
$$

where $\boldsymbol{H}$ denotes the magnetic field, $\boldsymbol{B}$ the magnetic field density (induction), $\boldsymbol{J}$ the electric current density, and $\boldsymbol{E}$ the electric field, respectively.

These equations are joined by the material relations

$$
\begin{aligned}
\boldsymbol{B} & =\mu \boldsymbol{H}, \\
\boldsymbol{J} & =\sigma \boldsymbol{E},
\end{aligned}
$$

with the magnetic permeability $\mu$ and the electric conductivity $\sigma$. Although in general $\mu$ and $\sigma$ are tensors, they are scalar in our case, since we consider only isotropic materials.

Whereas the conductivity can usually be assumed to be constant for each material, the relation between $\boldsymbol{B}$ and $\boldsymbol{H}$ is generally nonlinear. Disregarding the effects of hysteresis, we have $\boldsymbol{H}=\nu(|\boldsymbol{B}|) \boldsymbol{B}$, where the continuous function $\nu: \mathbb{R}_{0}^{+} \rightarrow \mathbb{R}^{+}$satisfies the following properties, which are immediate consequences of the physical background (cf. $[19,20])$ :

$$
\begin{gathered}
0<\underline{\nu} \leq \nu(s) \leq \bar{\nu}, \quad \forall s, \\
s \mapsto \nu(s) s \text { is strictly monotone, } \\
s \mapsto \nu(s) s \text { is Lipschitz continuous. }
\end{gathered}
$$

Introducing a vector potential $\boldsymbol{u}$ for the magnetic field density $\boldsymbol{B}=\operatorname{curl} \boldsymbol{u}$, we transform equation (1) to

$$
\sigma \frac{\partial \boldsymbol{u}}{\partial t}+\operatorname{curl}(\nu(|\operatorname{curl} \boldsymbol{u}|) \operatorname{curl} \boldsymbol{u})=\boldsymbol{f},
$$

where $f$ denotes the impressed and source currents.

In this section, we want to prove existence of a weak solution of problem (5) together with appropriate boundary conditions and an initial condition. Furthermore, we show that the solution is unique in a certain sense. This section is arranged in two parts: Firstly, we show that the problem is uniquely solvable in both conducting and non-conducting regions. Secondly, we conclude that the eddy current problem (5) is uniquely solvable in general domains consisting of non-conducting and conducting regions. 


\subsection{Some Results for Conducting and Non-Conducting Regions}

In electromagnetic problems, one often deals with conducting $(\sigma>0)$ and nonconducting $(\sigma=0)$ regions, for example in the case of some conducting part surrounded by air. The problem that we consider has a significantly different structure in these two cases. For non-conducting regions, equation (5) is a stationary and elliptic problem:

$$
\begin{aligned}
& \text { Find } \boldsymbol{u}(\boldsymbol{x}) \text { : } \\
& \operatorname{curl}(\nu \operatorname{curl} u)=f, \quad \text { in } \Omega, \\
& \boldsymbol{u} \times \boldsymbol{n}=\boldsymbol{g}, \quad \text { on } \Gamma \text {. }
\end{aligned}
$$

For conductors, on the other hand, we face a parabolic problem:

$$
\begin{array}{llll}
\text { Find } \boldsymbol{u}(\boldsymbol{x}, t) \text { : } & & & \\
\begin{aligned}
\sigma \frac{\partial \boldsymbol{u}}{\partial t}+\operatorname{curl}(\nu \operatorname{curl} \boldsymbol{u}) & =\boldsymbol{f}, & & \text { in } \Omega \times[0, T], \\
\boldsymbol{u} \times \boldsymbol{n} & =\boldsymbol{g}, & & \text { on } \Gamma \times[0, T], \\
\boldsymbol{u} & =\boldsymbol{u}_{0}, & & \text { on } \Omega \times\{0\} .
\end{aligned}
\end{array}
$$

We remark that in general the relation between the magnetic field $\boldsymbol{H}$ and the induction $\boldsymbol{B}$ could be nonlinear in both conducting and non-conducting regions. Mostly, however, for the non-conducting part we have a linear relation between $\boldsymbol{B}$ and $\boldsymbol{H}$, so there $\nu=\nu(\boldsymbol{x})$.

In the conducting region, $\nu(|\operatorname{curl} \boldsymbol{u}|)$ with the properties (2) and (3) is supposed to be given.

We will now prove that both problems are solvable and that the solution is unique in a certain sense. For this task, we consider the weak formulation of these equations.

\section{Non-Conducting Regions}

The weak formulation of (6) yields the variational equation

$$
\underbrace{\int_{\Omega} \nu \operatorname{curl} \boldsymbol{u} \cdot \operatorname{curl} \boldsymbol{v} \mathrm{d} \boldsymbol{x}}_{=: a(\boldsymbol{u}, \boldsymbol{v})}=\underbrace{\int_{\Omega} \boldsymbol{f} \boldsymbol{v} \mathrm{d} \boldsymbol{x}}_{=:\langle F, \boldsymbol{v}\rangle}, \quad \forall \boldsymbol{v} \in \boldsymbol{V}
$$

with $\boldsymbol{V}=\boldsymbol{H}_{0}(\operatorname{curl}, \Omega)=\{\boldsymbol{v} \in \boldsymbol{H}(\operatorname{curl}, \Omega): \boldsymbol{v} \times \boldsymbol{n}=0$ on $\Gamma\}$.

We are looking for a solution $\boldsymbol{u}$ in the linear manifold $\tilde{\boldsymbol{g}}+\boldsymbol{V}$, where $\tilde{\boldsymbol{g}} \in \boldsymbol{H}$ (curl) should meet the boundary condition $\tilde{\boldsymbol{g}} \times \boldsymbol{n}=\boldsymbol{g}$ on $\Gamma$. This is possible for $\boldsymbol{g} \in \boldsymbol{H}^{-\frac{1}{2}}\left(\operatorname{div}_{\Gamma}, \Gamma\right)$, since the tangential trace $\gamma_{\tau}: \boldsymbol{H}(\mathbf{c u r l}, \Omega) \rightarrow \boldsymbol{H}^{-\frac{1}{2}}\left(\operatorname{div}_{\Gamma}, \Gamma\right)$ is surjective [7].

Homogenization of the problem leads to

$$
\text { Find } \boldsymbol{u} \in \boldsymbol{V}: \quad a(\boldsymbol{u}, \boldsymbol{v})=\langle\tilde{F}, \boldsymbol{v}\rangle, \quad \forall \boldsymbol{v} \in \boldsymbol{V},
$$


with $\langle\tilde{F}, \boldsymbol{v}\rangle:=\langle F, \boldsymbol{v}\rangle-a(\tilde{\boldsymbol{g}}, \boldsymbol{v})$. The linear form $F \in \boldsymbol{V}^{*}$ and the bilinear form $a(\cdot, \cdot): \boldsymbol{H}($ curl $) \times \boldsymbol{H}($ curl $) \rightarrow \mathbb{R}$ are those defined in (8).

For gradient fields $\boldsymbol{v}$, the left hand side of $(8)=(9)$ equals zero, so clearly the source $f$ has to be weakly divergence-free in order to ensure solvability. This means $\int_{\Omega} \boldsymbol{f} \cdot \operatorname{grad} \phi \mathrm{d} \boldsymbol{x}=0$, for all $\phi \in H_{0}^{1}(\Omega)$, is a necessary condition for existence of a solution.

Remark 1. Later on, we will consider only divergence-free test functions. With this reduced set of test functions, no additional conditions for solvability are required, since the space $\boldsymbol{V}$ then does not contain any gradient fields.

The following considerations motivate this reduction to solenoidal functions: Since the curl of a gradient field vanishes, for every solution $\boldsymbol{u}$ of (9), we get a set of solutions $\boldsymbol{u}+\operatorname{grad} \phi, \phi \in H_{0}^{1}(\Omega)$, i.e. the problem is not uniquely solvable. However, we can show uniqueness in the factor space of divergence-free, i.e. solenoidal functions.

For multiply connected domains, we denote the $p \geq 1$ components of the boundary $\partial \Omega$ by $\Gamma_{i}, 1 \leq i \leq p$. In this general case, equation (9) does not change if we add $\operatorname{grad} \phi$ for arbitrary $\phi \in H^{1}(\Omega)$ with $\phi$ constant on each boundary component, i.e. $\phi=c_{i}$ on $\Gamma_{i}, 1 \leq i \leq p$.

This gives rise to the definition

$$
\begin{aligned}
\boldsymbol{W}(\Omega):=\boldsymbol{W}:= & \{\boldsymbol{w}=\operatorname{grad} \phi: \\
& \left.\phi \in H^{1}(\Omega) \text { and } \phi=c_{i} \text { on } \Gamma_{i}, 1 \leq i \leq p\right\} .
\end{aligned}
$$

For proving uniqueness, we factor the space $\boldsymbol{V}$ by $\boldsymbol{W}$, and restrict equation (9) to this factor space

$$
\overline{\boldsymbol{V}}:=\boldsymbol{V} / \boldsymbol{W} \simeq\left\{\boldsymbol{v} \in \boldsymbol{V}:(\boldsymbol{v}, \boldsymbol{w})_{\boldsymbol{L}_{2}}=0, \forall \boldsymbol{w} \in \boldsymbol{W}\right\} .
$$

So the problem that we deal with now reads as follows:

$$
\text { Find } \boldsymbol{u} \in \overline{\boldsymbol{V}}: \quad a(\boldsymbol{u}, \boldsymbol{v})=\langle\tilde{F}, \boldsymbol{v}\rangle, \quad \forall \boldsymbol{v} \in \overline{\boldsymbol{V}} .
$$

We will see that this variational problem is uniquely solvable by the Lax-Milgram theorem (e.g. [12]), or by Browder-Minty (e.g. [27]) in the nonlinear case. For proving existence and uniqueness, we need an important result on norm equivalence in the space $\boldsymbol{H}(\mathbf{c u r l}, \Omega) \cap \boldsymbol{H}(\operatorname{div}, \Omega)$ for multiply connected domains $\Omega$ :

Lemma 2 ([1]). Let $\Omega$ be multiply connected with Lipschitz boundary and boundary components $\Gamma_{i}, 1 \leq i \leq p$, let $X(\Omega):=\boldsymbol{H}(\mathbf{c u r l}, \Omega) \cap \boldsymbol{H}(\operatorname{div}, \Omega)$ with the norm

$$
\|\boldsymbol{v}\|_{X(\Omega)}^{2}=\|\boldsymbol{v}\|_{\boldsymbol{L}_{2}(\Omega)}^{2}+\|\operatorname{curl} \boldsymbol{v}\|_{\boldsymbol{L}_{2}(\Omega)}^{2}+\|\operatorname{div} \boldsymbol{v}\|_{L_{2}(\Omega)}^{2} .
$$

Then on the space $X_{N}(\Omega)=\{\boldsymbol{v} \in X(\Omega): \boldsymbol{v} \times \boldsymbol{n}=0$ on $\partial \Omega\}$, the seminorm

$$
\boldsymbol{v} \mapsto\|\operatorname{curl} \boldsymbol{v}\|_{\boldsymbol{L}_{2}(\Omega)}+\|\operatorname{div} \boldsymbol{v}\|_{L_{2}(\Omega)}+\sum_{i=1}^{p}\left|\langle\boldsymbol{v} \cdot \boldsymbol{n}, 1\rangle_{\Gamma_{i}}\right|
$$

is equivalent to the norm $\|\cdot\|_{X(\Omega)}$. 
Remark 3. As can easily be seen, in the space $\overline{\boldsymbol{V}}$ both div $\boldsymbol{v}$ in the domain and $\boldsymbol{v} \cdot \boldsymbol{n}$ on each boundary component are zero: By the definition of $\overline{\boldsymbol{V}}=\boldsymbol{V} / \boldsymbol{W}$, we have $\int_{\Omega} \boldsymbol{v} \cdot \operatorname{grad} \phi \mathrm{d} \boldsymbol{x}=0$ for all functions $\phi \in H^{1}(\Omega)$ that are constant on all components of the boundary. Integration by parts yields

$$
-\int_{\Omega} \operatorname{div} \boldsymbol{v} \cdot \phi \mathrm{d} \boldsymbol{x}+\sum_{i} c_{i}(\phi) \int_{\Gamma_{i}} \boldsymbol{v} \cdot \boldsymbol{n} \mathrm{ds}=0 .
$$

Since this equality is satisfied for all $\phi \in H_{0}^{1}(\Omega)$, div $\boldsymbol{v}$ equals zero almost everywhere, and thus also $\boldsymbol{v} \cdot \boldsymbol{n}$ on each boundary component.

Consequently, Lemma 2 provides the required equivalence between the full $\boldsymbol{H}(\mathbf{c u r l})$ norm and the seminorm $|\boldsymbol{u}|^{2}=\int_{\Omega}|\operatorname{curl} \boldsymbol{u}|^{2}$.

Throughout the rest of this paper we assume $\Omega$ to be multiply connected with Lipschitz boundary.

Lemma 4. For $\nu \in L_{\infty}(\Omega)$ with $0<\underline{\nu} \leq \nu(\boldsymbol{x}) \leq \bar{\nu}$ almost everywhere (a.e.) in $\Omega$, there exists a unique $\boldsymbol{u} \in \overline{\boldsymbol{V}}$ solving

$$
a(\boldsymbol{u}, \boldsymbol{v})=\langle\tilde{F}, \boldsymbol{v}\rangle, \quad \forall \boldsymbol{v} \in \overline{\boldsymbol{V}}
$$

Proof. By the Lax-Milgram theorem, it suffices to show that $a(\cdot, \cdot)$ is bilinear, $\overline{\boldsymbol{V}}$-elliptic and $\overline{\boldsymbol{V}}$-continuous and that $\tilde{F} \in \overline{\boldsymbol{V}}^{*}$.

Bilinearity is evident, the same holds for linearity of $\tilde{F} . \overline{\boldsymbol{V}}$-ellipticity and $\overline{\boldsymbol{V}}$ continuity of $a(\cdot, \cdot)$ follow immediately from the assumptions on $\nu$ and the norm equivalence stated in Lemma 2 and Remark 3. This norm equivalence also implies the boundedness of $\tilde{F}$.

Remark 5. Problem (12) is uniquely solvable for nonlinear reluctivity $\nu=$ $\nu(|\operatorname{curl} \boldsymbol{u}|)$ as well, if $\nu$ fulfills the assumptions (2) and (3). Existence and uniqueness then follow by Browder's and Minty's theorem or by the nonlinear version of Lax-Milgram with the additional condition (4).

\section{Conducting Regions}

We continue by analyzing the parabolic problem in the conducting area:

The weak formulation of (7) yields the variational equation

$$
\int_{\Omega} \sigma \frac{\partial \boldsymbol{u}}{\partial t} \boldsymbol{v} \mathrm{d} \boldsymbol{x}+\underbrace{\int_{\Omega} \nu(|\operatorname{curl} \boldsymbol{u}|) \operatorname{curl} \boldsymbol{u} \cdot \operatorname{curl} \boldsymbol{v} \mathrm{d} \boldsymbol{x}}_{=:\langle A(\boldsymbol{u}), \boldsymbol{v}\rangle}=\underbrace{\int_{\Omega} \boldsymbol{f} \boldsymbol{v} \mathrm{d} \boldsymbol{x}}_{=:\langle F, \boldsymbol{v}\rangle}
$$

for all $\boldsymbol{v} \in \boldsymbol{V}$ and almost everywhere in $(0, T)$, with $\boldsymbol{V}=\boldsymbol{H}_{0}(\operatorname{curl}, \Omega)=$ $\{\boldsymbol{v} \in \boldsymbol{H}(\operatorname{curl}, \Omega): \boldsymbol{v} \times \boldsymbol{n}=0$ on $\Gamma\}$ as above.

We homogenize the problem as before. Furthermore, we also restrict the equation to the factor space $\overline{\boldsymbol{V}}$ as defined in (11), at least at first. Later on, we 
will see that the solution is unique in $\boldsymbol{V}=\boldsymbol{H}_{0}$ (curl), not only in the space of weakly divergence-free functions $\overline{\boldsymbol{V}}=\boldsymbol{V} / \boldsymbol{W}(\Omega)$.

So, almost everywhere in $(0, T)$, the problem we are concerned with reads as follows:

$$
\text { Find } \boldsymbol{u} \in \overline{\boldsymbol{V}}:\left(\sigma \frac{\partial \boldsymbol{u}}{\partial t}, \boldsymbol{v}\right)_{\boldsymbol{L}_{2}}+\langle A(\boldsymbol{u}), \boldsymbol{v}\rangle=\langle\tilde{F}, \boldsymbol{v}\rangle, \quad \forall \boldsymbol{v} \in \overline{\boldsymbol{V}}
$$

Here, $A: \overline{\boldsymbol{V}} \rightarrow \overline{\boldsymbol{V}}^{*}$ is the operator defined in (13), and $\tilde{F}$ results from the homogenization: If we have $\tilde{\boldsymbol{g}}(t)$ fulfilling the boundary conditions, we get $\langle\tilde{F}, \boldsymbol{v}\rangle:=\langle F, \boldsymbol{v}\rangle-\left(\sigma \frac{\partial \tilde{\boldsymbol{g}}}{\partial t}, \boldsymbol{v}\right)_{\boldsymbol{L}_{2}}-\langle A(\tilde{\boldsymbol{g}}), \boldsymbol{v}\rangle$ with $F$ as in $(13)$.

Let us now consider $\boldsymbol{u}$ as a function $\boldsymbol{u}:[0, T] \rightarrow \overline{\boldsymbol{V}}, t \mapsto \boldsymbol{u}(\cdot, t)$ and define the operators $\bar{A}: L_{2}((0, T), \overline{\boldsymbol{V}}) \rightarrow L_{2}\left((0, T), \overline{\boldsymbol{V}}^{*}\right)$ and $\bar{F} \in L_{2}\left((0, T), \overline{\boldsymbol{V}}^{*}\right)$ as follows:

$$
\begin{aligned}
\langle\bar{A}(\boldsymbol{u})(t), \boldsymbol{v}\rangle:= & \int_{\Omega} \nu(|\operatorname{curl} \boldsymbol{u}(t)|) \operatorname{curl} \boldsymbol{u}(t) \cdot \operatorname{curl} \boldsymbol{v} \mathrm{d} \boldsymbol{x}, \\
& \forall \boldsymbol{v} \in \overline{\boldsymbol{V}}, \boldsymbol{u} \in L_{2}((0, T), \overline{\boldsymbol{V}}), \\
\langle\bar{F}(t), \boldsymbol{v}\rangle:= & \int_{\Omega} \boldsymbol{f}(t) \cdot \boldsymbol{v} \mathrm{d} \boldsymbol{x}-\int_{\Omega} \sigma \frac{\partial \tilde{\boldsymbol{g}}(t)}{\partial t} \cdot \boldsymbol{v} \mathrm{d} \boldsymbol{x}-\langle\bar{A}(\tilde{\boldsymbol{g}})(t), \boldsymbol{v}\rangle, \\
& \forall \boldsymbol{v} \in \overline{\boldsymbol{V}} .
\end{aligned}
$$

Here we require $\tilde{\boldsymbol{g}} \in L_{2}((0, T), \boldsymbol{H}(\mathbf{c u r l}))$ with its time derivative $\frac{\partial \tilde{\boldsymbol{g}}}{\partial t} \in L_{2}\left((0, T), \boldsymbol{H}(\mathbf{c u r l})^{*}\right)$ for the definition of $\bar{F}$. This prerequisite is met by any reasonable function $\boldsymbol{g}(t)$ in the boundary condition, e.g. for $\boldsymbol{g} \in L_{2}\left((0, T), \boldsymbol{H}^{-\frac{1}{2}}\left(\operatorname{div}_{\Gamma}, \Gamma\right)\right)$ with $\frac{\partial \boldsymbol{g}}{\partial t} \in L_{2}\left((0, T), \boldsymbol{H}^{-\frac{1}{2}}\left(\operatorname{div}_{\Gamma}, \Gamma\right)\right)$.

With the above definitions, equation (14) together with the initial condition $\boldsymbol{u}(\boldsymbol{x}, 0)=\boldsymbol{u}_{0}(\boldsymbol{x})$, i.e. the original equation (7) in its weak formulation, can be written as an operator equation in $L_{2}\left((0, T), \overline{\boldsymbol{V}}^{*}\right)$ :

Find $\boldsymbol{u} \in L_{2}((0, T), \overline{\boldsymbol{V}})$ with $\dot{\boldsymbol{u}} \in L_{2}\left((0, T), \overline{\boldsymbol{V}}^{*}\right)$ such that

$$
\begin{aligned}
\sigma \dot{\boldsymbol{u}}+\bar{A}(\boldsymbol{u}) & =\bar{F}, \\
\boldsymbol{u}(0) & =\boldsymbol{u}_{0},
\end{aligned}
$$

with $\boldsymbol{u}_{0} \in \boldsymbol{L}_{2}(\Omega)$.

Note that the initial condition $(17 \mathrm{~b})$ is meaningful, because the Banach space valued functions $\left\{\boldsymbol{u} \in L_{2}((0, T), \overline{\boldsymbol{V}}): \dot{\boldsymbol{u}} \in L_{2}\left((0, T), \overline{\boldsymbol{V}}^{*}\right)\right\}$ can be continuously embedded into $C\left([0, T], L_{2}(\Omega)\right)$, see $[26]$.

For proving unique solvability of (17), we first quote an important theorem on existence and uniqueness of solutions of nonlinear parabolic problems, which can be found e.g. in [27]: 
Theorem 6. Let $V \subset H \subset V^{*}$ be an evolution triple and let $A: V \rightarrow V^{*}$ be a hemicontinuous, monotone and coercive operator. Suppose furthermore that $A$ is bounded, and let $u_{0} \in H$ and $b \in L_{2}\left((0, T), V^{*}\right)$ (with $\left.0<T<\infty\right)$ be given. Then the initial value problem

$$
\begin{gathered}
u^{\prime}(t)+A(u(t))=b(t), \quad \text { for almost all } t \in(0, T), \\
u(0)=u_{0} \in H, \\
u \in L_{2}((0, T), V), u^{\prime} \in L_{2}\left((0, T), V^{*}\right),
\end{gathered}
$$

has a unique solution.

Lemma 7. Let $s \mapsto \nu(s)$ be continuous, $0<\underline{\nu} \leq \nu(s) \leq \bar{\nu}, \forall s \in \mathbb{R}_{0}^{+}$and let the function $s \mapsto \nu(s) s$ be monotone.

Suppose $\sigma \in L_{\infty}$ to be strictly positive and let moreover $\boldsymbol{u}_{0} \in \boldsymbol{L}_{2}(\Omega), \boldsymbol{f} \in$ $L_{2}\left((0, T), \overline{\boldsymbol{V}}^{*}\right)$ and the inhomogeneity $\tilde{\boldsymbol{g}} \in L_{2}((0, T), \boldsymbol{H}(\mathbf{c u r l}))$ with $\frac{\partial \tilde{\boldsymbol{g}}}{\partial t} \in L_{2}\left((0, T), \boldsymbol{H}(\mathbf{c u r l})^{*}\right)$ be given.

Then we have a unique $\boldsymbol{u} \in L_{2}((0, T), \overline{\boldsymbol{V}})$ with $\dot{\boldsymbol{u}} \in L_{2}\left((0, T), \overline{\boldsymbol{V}}^{*}\right)$ which solves (17), i.e.

$$
\begin{aligned}
\sigma \dot{\boldsymbol{u}}+\bar{A}(\boldsymbol{u}) & =\bar{F} \\
\boldsymbol{u}(0) & =\boldsymbol{u}_{0}
\end{aligned}
$$

Proof. It is easy to see that - due to the norm equivalence in $\overline{\boldsymbol{V}}$ (Lemma 2) and the assumptions on $\nu$ - the prerequisites of Theorem 6 are fulfilled.

Uniqueness in $\boldsymbol{H}_{0}(\mathbf{c u r l})$ : Our next issue is to show that under certain assumptions the solution given by Lemma 7 is unique in the whole space $\boldsymbol{V}=$ $\boldsymbol{H}_{0}(\mathbf{c u r l})$. For this task we consider equation (17) with an arbitrary test function $\boldsymbol{w} \in \boldsymbol{W}$ :

$$
\begin{aligned}
\int_{\Omega} \sigma \frac{\partial \boldsymbol{u}}{\partial t} & \boldsymbol{w} \mathrm{d} \boldsymbol{x}+\int_{\Omega} \nu(|\operatorname{curl} \boldsymbol{u}|) \operatorname{curl} \boldsymbol{u} \cdot \operatorname{curl} \boldsymbol{w} \mathrm{d} \boldsymbol{x} \\
& =\int_{\Omega} \boldsymbol{f} \boldsymbol{w} \mathrm{d} \boldsymbol{x}-\int_{\Omega} \sigma \frac{\partial \tilde{\boldsymbol{g}}}{\partial t} \boldsymbol{w} \mathrm{d} \boldsymbol{x}-\int_{\Omega} \nu(|\operatorname{curl} \tilde{\boldsymbol{g}}|) \operatorname{curl} \boldsymbol{u} \cdot \operatorname{curl} \boldsymbol{w} \mathrm{d} \boldsymbol{x} .
\end{aligned}
$$

Suppose now, that $\boldsymbol{f}(t)$ is divergence-free for all $t$, i.e.

$$
\int_{\Omega} \boldsymbol{f}(t) \cdot \boldsymbol{w}=0, \quad \forall t \in[0, T], \forall \boldsymbol{w} \in \boldsymbol{W},
$$

where $\boldsymbol{W}=\left\{\boldsymbol{w}=\operatorname{grad} \phi: \phi \in H^{1}(\Omega)\right.$ and $\phi=c_{i}$ on $\left.\Gamma_{i}, 1 \leq i \leq p\right\}$ as defined in (10). Assume moreover that the same holds for $\sigma \frac{\partial \tilde{g}}{\partial t}(t)$. Then the right hand side in (19) equals zero, what implies

$$
\int_{\Omega} \sigma \frac{\partial \boldsymbol{u}}{\partial t} \boldsymbol{w}=0, \quad \forall \boldsymbol{w} \in \boldsymbol{W},
$$


since the curl of a gradient field $\boldsymbol{w} \in \boldsymbol{W}$ vanishes.

In other words, for constant conductivity $\sigma$, the time derivative of the function we are looking for is divergence-free for all moments in time. This means that provided the initial solution $\boldsymbol{u}_{0}$ is solenoidal as well - all possible solutions $\boldsymbol{u}(t)$ are divergence-free and thus lie in $\overline{\boldsymbol{V}}$, where we have already shown existence and uniqueness in Lemma 7.

We summarize these ideas in the following lemma:

Lemma 8. Suppose that the functions $\boldsymbol{f}(t)$ and $\sigma \frac{\partial \tilde{\boldsymbol{g}}}{\partial t}(t)$ are defined for all $t \in$ $[0, T]$ and divergence-free in the following weak sense:

$$
\begin{aligned}
\int_{\Omega} \boldsymbol{f}(t) \cdot \boldsymbol{w} & =0, & \forall t \in[0, T], \forall \boldsymbol{w} \in \boldsymbol{W}, \\
\int_{\Omega} \sigma \frac{\partial \tilde{\boldsymbol{g}}}{\partial t}(t) \cdot \boldsymbol{w} & =0, & \forall t \in[0, T], \forall \boldsymbol{w} \in \boldsymbol{W} .
\end{aligned}
$$

Assume moreover that $\sigma \boldsymbol{u}_{0}$ is divergence-free as well, i.e.

$$
\int_{\Omega} \sigma \boldsymbol{u}_{0} \cdot \boldsymbol{w}=0, \quad \forall \boldsymbol{w} \in \boldsymbol{W}
$$

Then, if there is a solution $\boldsymbol{u}$ of (17), we have

$$
\int_{\Omega} \sigma \boldsymbol{u}(t) \cdot \boldsymbol{w}=0, \quad \forall t \in[0, T], \forall \boldsymbol{w} \in \boldsymbol{W}
$$

Proof. We have already shown this in the discussion preceding this lemma.

Remark 9. We emphasize again that any solution of (17) is defined for all $t$ and continuous because of the embedding $\left\{\boldsymbol{u} \in L_{2}((0, T), \overline{\boldsymbol{V}}): \dot{\boldsymbol{u}} \in L_{2}\left((0, T), \overline{\boldsymbol{V}}^{*}\right)\right\} \subset$ $C\left([0, T], L_{2}(\Omega)\right)$.

For constant conductivity in the whole domain $\Omega$, we immediately get the following consequence:

Corollary 10. Let the assumptions of the Lemmata 7 and 8 be satisfied. Suppose furthermore that $\sigma=$ const.

Then there exists a unique $\boldsymbol{u} \in L_{2}((0, T), \boldsymbol{V})$ with $\dot{\boldsymbol{u}} \in L_{2}\left((0, T), \boldsymbol{V}^{*}\right)$ such that

$$
\begin{aligned}
\sigma \dot{\boldsymbol{u}}+\bar{A}(\boldsymbol{u}) & =\bar{F}, \\
\boldsymbol{u}(0) & =\boldsymbol{u}_{0},
\end{aligned}
$$

and this solution $\boldsymbol{u}$ is divergence-free for all $t$.

\subsection{Application to Eddy Current Problems}

Taking advantage of the knowledge we have gained in the previous paragraphs, we can now prove the main result of this section, to wit existence of a solution of the eddy current problem and its uniqueness in a certain sense. 
The Lemmata 4 and 7 provide existence and uniqueness of the solution in nonconducting and conducting regions, respectively. However, we have not treated the case of domains consisting of parts with positive conductivity and of regions with $\sigma=0$ yet.

Usually, the domain of the whole eddy current problem (5) is such a mixed domain, because we often face a conducting region $\Omega_{1}$ together with some surrounding air $\Omega_{2}=\Omega \backslash \bar{\Omega}_{1}$, where we have $\sigma=0$. We sketch the situation of a simple two-dimensional problem in Figure 1.

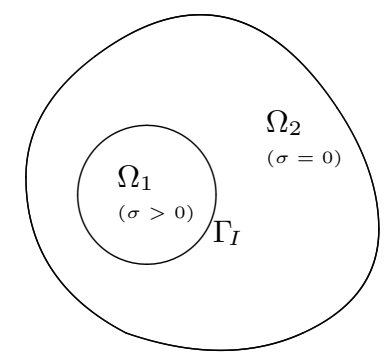

Figure 1: Sketch of the domain $\bar{\Omega}=\bar{\Omega}_{1} \cup \bar{\Omega}_{2}$.

Consequently, our task in the following paragraph consists in assembling the previous results to the desired theorem. The main idea for the proof is a Schur complement approach based on the observation that the solution in the nonconducting part is uniquely determined by the solution in the conducting region.

Let us first consider some fixed moment in time $t$. For joining the solutions in the respective domains, we need an interface condition on $\Gamma_{I}=\bar{\Omega}_{1} \cap \bar{\Omega}_{2}$ : The tangential component of the joined solution should be continuous, i.e. we should have

$$
\boldsymbol{u}_{1} \times \boldsymbol{n}_{1}=-\boldsymbol{u}_{2} \times \boldsymbol{n}_{2}, \quad \text { on } \Gamma_{I},
$$

where $\boldsymbol{u}_{1}$ and $\boldsymbol{u}_{2}$ are the solutions in $\Omega_{1}$ and $\Omega_{2}$ at the fixed time $t$, and $\boldsymbol{n}_{i}$ are the respective outer unit normal vectors at the interface $\Gamma_{I}$.

Suppose we knew the solution $\boldsymbol{u}_{1}$ in the conducting region. Then the interface condition (21) together with the original boundary condition

$$
\boldsymbol{u} \times \boldsymbol{n}=0, \quad \text { on } \Gamma=\partial \Omega,
$$

provide the necessary boundary conditions for the equation in the non-conducting part (6). So by Lemma 4 we have a unique divergence-free solution $\boldsymbol{u}_{2} \in \tilde{\boldsymbol{g}}+\overline{\boldsymbol{V}}_{2}$, where $\tilde{\boldsymbol{g}} \in \boldsymbol{H}\left(\mathbf{c u r l}, \Omega_{2}\right)$ satisfies the boundary conditions, and $\overline{\boldsymbol{V}}_{2}$ is the factor space of divergence-free functions as in (11). In other words, the solution in the conducting domain $\Omega_{1}$ uniquely determines the solution in the non-conducting region $\Omega_{2}$.

We summarize these considerations in the following lemma: 
Lemma 11. Let $\bar{\Omega}=\bar{\Omega}_{1} \cup \bar{\Omega}_{2}$ with $\Omega_{1} \cap \Omega_{2}=\emptyset$ and let $\Omega_{2}$ be multiply connected with Lipschitz boundary. Assume $\nu \in L_{\infty}\left(\Omega_{2}\right)$ with $0<\underline{\nu} \leq \nu(\boldsymbol{x}) \leq \bar{\nu}$ a.e. in $\Omega_{2}$.

Let $\boldsymbol{V}=\left\{\boldsymbol{v} \in \boldsymbol{H}\left(\operatorname{curl}, \Omega_{2}\right): \boldsymbol{v} \times \boldsymbol{n}=0\right.$ on $\left.\partial \Omega_{2}\right\}$ and $\boldsymbol{W}=\boldsymbol{W}\left(\Omega_{2}\right)$ as in (10). Define $\overline{\boldsymbol{V}}:=\boldsymbol{V} / \boldsymbol{W}$ and let $\boldsymbol{f} \in \overline{\boldsymbol{V}}^{*}$ be given.

Then for each $\boldsymbol{u}_{1} \in \boldsymbol{H}\left(\mathbf{c u r l}, \Omega_{1}\right)$ exists exactly one function $\boldsymbol{u}_{2} \in \tilde{\boldsymbol{g}}+\overline{\boldsymbol{V}}$ such that $\boldsymbol{u}_{2}$ is the weak solution of

$$
\begin{aligned}
\operatorname{curl}\left(\nu \operatorname{curl} \boldsymbol{u}_{2}\right) & =\boldsymbol{f}, & & \text { in } \Omega_{2}, \\
\boldsymbol{u}_{2} \times \boldsymbol{n} & =0, & & \text { on } \partial \Omega \cap \partial \Omega_{2}, \\
\boldsymbol{u}_{2} \times \boldsymbol{n} & =\boldsymbol{u}_{1} \times \boldsymbol{n}, & & \text { on } \partial \Omega_{1} \cap \partial \Omega_{2},
\end{aligned}
$$

where $\boldsymbol{n}$ is the outer unit normal vector to $\partial \Omega_{2}$ and $\tilde{\boldsymbol{g}} \in \boldsymbol{H}\left(\mathbf{c u r l}, \Omega_{2}\right)$ satisfies the boundary conditions.

Lemma 11 shows that $\boldsymbol{u}_{2}=\boldsymbol{U}\left(\boldsymbol{u}_{1}\right)$ for some function $\boldsymbol{U}: \boldsymbol{H}\left(\operatorname{curl}, \Omega_{1}\right) \rightarrow$ $\boldsymbol{H}\left(\operatorname{curl}, \Omega_{2}\right)$, i.e. the solution in $\Omega_{2}$ is uniquely defined by the solution in $\Omega_{1}$. We point out that the eddy current problem is uniquely solvable in the conducting region $\Omega_{1}$ (Lemma 7 ). Consequently, the idea to take advantage of the relation $\boldsymbol{u}_{2}=\boldsymbol{U}\left(\boldsymbol{u}_{1}\right)$ for proving unique solvability of the whole eddy current problem seems obvious.

For our proof, we define a space $\tilde{\boldsymbol{V}}$ which contains arbitrary divergence-free functions in $\Omega_{1}$, but only those functions in the nonconducting domain $\Omega_{2}$ that are determined by the function $\boldsymbol{U}: \boldsymbol{H}\left(\mathbf{c u r l}, \Omega_{1}\right) \rightarrow \boldsymbol{H}\left(\mathbf{c u r l}, \Omega_{2}\right)$. Moreover, we impose homogeneous Dirichlet boundary conditions as usual:

$$
\begin{aligned}
\tilde{\boldsymbol{V}}:=\{\boldsymbol{v} \in \boldsymbol{H}(\operatorname{curl}, \Omega): & \boldsymbol{v}_{\left.\right|_{\Omega_{1}}} \in \boldsymbol{H}\left(\operatorname{curl}, \Omega_{1}\right), \\
& \boldsymbol{v}_{\left.\right|_{\Omega_{2}}}=\boldsymbol{U}\left(\boldsymbol{v}_{\left.\right|_{\Omega_{1}}}\right), \\
& (\boldsymbol{v}, \boldsymbol{w})_{\boldsymbol{L}_{2}}=0, \forall \boldsymbol{w} \in \boldsymbol{W}\left(\Omega_{1}\right), \\
& \boldsymbol{v} \times \boldsymbol{n}=0, \text { on } \Gamma=\partial \Omega\},
\end{aligned}
$$

where $\boldsymbol{W}\left(\Omega_{1}\right)$ are the gradient fields on $\Omega_{1}$ as defined in (10).

The space $\tilde{\boldsymbol{V}}$ is just another representation of the divergence-free $\boldsymbol{H}$ (curl)functions in $\Omega_{1}$ with zero tangential component on the boundary $\tilde{\Gamma}:=\partial \Omega \cap \partial \Omega_{1}$, i.e. $\tilde{\boldsymbol{V}} \simeq \boldsymbol{H}_{\tilde{\Gamma}}\left(\operatorname{curl}, \Omega_{1}\right) / \boldsymbol{W}\left(\Omega_{1}\right)$. Since in $\Omega_{1}$ we deal with the nonlinear parabolic problem (17), and since this equation is uniquely solvable by Lemma 7 , we may hope that the whole eddy current problem restricted to $\tilde{\boldsymbol{V}}$ is uniquely solvable as well. As we see in the following main theorem on unique solvability of eddy current problems, this is actually the case.

Theorem 12. Let $s \mapsto \nu_{1}(s)$ be continuous, $0<\underline{\nu}_{1} \leq \nu_{1}(s) \leq \bar{\nu}_{1}, \forall s \in \mathbb{R}_{0}^{+}$and let the function $s \mapsto \nu_{1}(s) s$ be monotone. Assume furthermore $\nu_{2} \in L_{\infty}\left(\Omega_{2}\right)$ with $0<\underline{\nu}_{2} \leq \nu_{2}(\boldsymbol{x}) \leq \bar{\nu}_{2}$ a.e. in $\Omega_{2}$. 
Let moreover $\boldsymbol{u}_{0} \in \boldsymbol{L}_{2}(\Omega)$ and $\boldsymbol{f} \in L_{2}\left((0, T), \tilde{\boldsymbol{V}}^{*}\right)$ be given, and suppose $\sigma \in L_{\infty}$ to be strictly positive.

Then there is a unique $\boldsymbol{u} \in L_{2}((0, T), \tilde{\boldsymbol{V}})$ with $\dot{\boldsymbol{u}} \in L_{2}\left((0, T), \tilde{\boldsymbol{V}}^{*}\right)$ such that $\boldsymbol{u}$ is the weak solution of (5), more precisely of

$$
\begin{aligned}
\sigma \frac{\partial \boldsymbol{u}}{\partial t}+\operatorname{curl}\left(\nu_{1}(|\operatorname{curl} \boldsymbol{u}|) \operatorname{curl} \boldsymbol{u}\right) & =\boldsymbol{f}, & & \text { in } \Omega_{1} \times[0, T], \\
\operatorname{curl}\left(\nu_{2} \operatorname{curl} \boldsymbol{u}\right) & =\boldsymbol{f}, & & \text { in } \Omega_{2} \times[0, T], \\
\boldsymbol{u} \times \boldsymbol{n} & =0, & & \text { on } \Gamma \times[0, T], \\
\boldsymbol{u} & =\boldsymbol{u}_{0}, & & \text { on } \Omega \times\{0\},
\end{aligned}
$$

with a continuos tangential component along the interface $\bar{\Omega}_{1} \cap \bar{\Omega}_{2}$.

This means that we have a unique $\boldsymbol{u} \in L_{2}((0, T), \tilde{\boldsymbol{V}})$ with $\dot{\boldsymbol{u}} \in$ $L_{2}\left((0, T), \tilde{\boldsymbol{V}}^{*}\right)$ solution of

$$
\begin{aligned}
\sigma \dot{\boldsymbol{u}}+A_{1}(\boldsymbol{u}) & =F, \quad \text { in } \Omega_{1}, \\
A_{2} \boldsymbol{u} & =F, \quad \text { in } \Omega_{2}, \\
\boldsymbol{u}_{0} & =\boldsymbol{u}_{0},
\end{aligned}
$$

with the operators $A_{1}, A_{2}$ and $F$ as in (8) and (13), respectively.

Proof. By the choice of the space $\tilde{\boldsymbol{V}}$, i.e. by Lemma 11, equation (24b) is fulfilled for any $\boldsymbol{u} \in L_{2}((0, T), \tilde{\boldsymbol{V}})$.

Remains to show that equation (24a) with the initial condition (24c) is uniquely solvable. We have already proven this fact in Lemma 7 for the space $\overline{\boldsymbol{V}}$ instead of $\tilde{\boldsymbol{V}}$. However, since $\tilde{\boldsymbol{V}}$ is nothing more than another representation of $\overline{\boldsymbol{V}}$, the result can be carried over to $\tilde{\boldsymbol{V}}$ one-to-one.

Thus we have proven that (24) is uniquely solvable.

Remark 13. We mention that no solvability condition for the source $\boldsymbol{f}$ is required (cf. Remark 1), since the space of test functions does not contain any gradient fields. Moreover, we stress that the condition $\boldsymbol{f} \in L_{2}\left((0, T), \tilde{\boldsymbol{V}}^{*}\right)$ is satisfied for any reasonable right hand side. For example, $\boldsymbol{f} \in L_{2}\left((0, T), \boldsymbol{L}_{2}(\Omega)\right)$ is clearly sufficient.

Under certain assumptions, the solution is not only unique among the divergencefree functions, but even in the space

$$
\begin{aligned}
\hat{\boldsymbol{V}}=\{\boldsymbol{v} \in \boldsymbol{H}(\operatorname{curl}, \Omega): & \boldsymbol{v}_{\left.\right|_{\Omega_{1}}} \in \boldsymbol{H}\left(\operatorname{curl}, \Omega_{1}\right), \\
& \boldsymbol{v}_{\left.\right|_{\Omega_{2}}}=\boldsymbol{U}\left(\boldsymbol{v}_{\left.\right|_{\Omega_{1}}}\right), \\
& \boldsymbol{v} \times \boldsymbol{n}=0, \text { on } \Gamma=\partial \Omega\} .
\end{aligned}
$$

This means the solution is unique among those $\boldsymbol{H}$ (curl)-functions that are divergence-free in the non-conducting region $\Omega_{2}$ and arbitrary in $\Omega_{1}$. We summarize this result, which is an immediate consequence of Theorem 12 and Corollary 10, in the following corollary: 
Corollary 14. Let the assumptions of Theorem 12 be satisfied and suppose that $\sigma=$ const. Let furthermore $\boldsymbol{f}(t)$ be defined for all $t$ and $\boldsymbol{f}(t)$ and $\boldsymbol{u}_{0}$ be divergence-free in $\Omega_{1}$, i.e.

$$
\begin{aligned}
\int_{\Omega_{1}} \boldsymbol{f}(t) \cdot \boldsymbol{w}=0, & \forall t \in[0, T], \forall \boldsymbol{w} \in \boldsymbol{W}\left(\Omega_{1}\right), \\
\int_{\Omega_{1}} \boldsymbol{u}_{0} \cdot \boldsymbol{w}=0, & \forall \boldsymbol{w} \in \boldsymbol{W}\left(\Omega_{1}\right) .
\end{aligned}
$$

Moreover, let $\hat{\boldsymbol{V}}$ be defined as in (25).

Then there exists a unique $\boldsymbol{u} \in L_{2}((0, T), \hat{\boldsymbol{V}})$ with $\dot{\boldsymbol{u}} \in L_{2}\left((0, T), \hat{\boldsymbol{V}}^{*}\right)$ solving (24), and this solution is divergence-free for all $t$.

\section{Truncated Fourier Series Expansion}

We recall that we are engaged in the solution of the problem

$$
\operatorname{curl}(\nu \operatorname{curl} \boldsymbol{u})+\sigma \frac{\partial \boldsymbol{u}}{\partial t}=\boldsymbol{f},
$$

with Dirichlet boundary conditions and an initial condition. In order to solve this equation numerically, we need a discretization in time and space. Instead of semi-discretizing the equation in space and solving the resulting ODE by a time-stepping method, we take advantage of the periodicity of the source current and thus the expected solution: Since the right hand side of (5) is a harmonic current of the form $\hat{f} \cdot \cos (\omega t)$, the ansatz

$$
\boldsymbol{u}(\boldsymbol{x}, t)=\boldsymbol{u}^{c}(\boldsymbol{x}) \cdot \cos (\omega t)+\boldsymbol{u}^{s}(\boldsymbol{x}) \cdot \sin (\omega t)
$$

seems to be manifest. This would reduce equation (5) to an equation for the coefficients $\boldsymbol{u}^{c}(\boldsymbol{x})$ and $\boldsymbol{u}^{s}(\boldsymbol{x})$ that only depend on the spatial coordinates.

In the linear case (i.e. for $\nu$ independent of $\operatorname{curl} \boldsymbol{u}$ ), the solution can be expressed in terms of the same base frequency $\omega$ as the given current source. Due to the nonlinearity, however, the solution depends on higher harmonics as well, but will still be periodical and can consequently be represented as a Fourier series. Truncating this series at some finite number $N$, i.e. applying a so-called multiharmonic ansatz, then yields a system of equations for the Fourier coefficients.

We mention that the Fourier series $\sum\left[\boldsymbol{u}_{k}^{c} \cos (k \omega t)+\boldsymbol{u}_{k}^{s} \sin (k \omega t)\right]$ can also be written in complex notation as

$$
\boldsymbol{u}(\boldsymbol{x}, t)=\operatorname{Re} \sum_{k=0}^{\infty} \hat{\boldsymbol{u}}_{k} \cdot e^{i k \omega t},
$$

with $\hat{\boldsymbol{u}}_{k}=\boldsymbol{u}_{k}^{c}-i \boldsymbol{u}_{k}^{s}$.

Since the projector Re is injective for Fourier series (26) with $\hat{\boldsymbol{u}}_{0}=0$, the problem can be regarded as a complex one. This means that the projector Re 
can be skipped without losing unique solvability (cf. [3]). Although in some publications (e.g. $[4,13,25])$ this fact is exploited for rewriting the problem as a system of complex equations, we prefer to stay with the formulation over $\mathbb{R}$, because the complex operator does not satisfy the Cauchy-Riemann equations and thus is not differentiable (e.g. $[13,16]$ ), what eliminates the possibility of linearization by the Newton method (cf. [3]).

\subsection{Steady State Solution}

In many eddy current problems, we are not so much interested in the response of some device to closure of the electric circuit, but more in its behavior under a harmonic current for the time $t \rightarrow \infty$. So what we really want to calculate is a steady state solution, i.e. a solution of the original problem (5) without the initial condition:

Definition 15. The function $\boldsymbol{u}(\boldsymbol{x}, t)$ is called a periodic steady state solution of equation (5), if

1. $\boldsymbol{u}$ satisfies (5) (but not necessarily the initial condition),

2. $\boldsymbol{u}$ is periodic, i.e. $\exists T \forall t: \boldsymbol{u}(\boldsymbol{x}, t)=\boldsymbol{u}(\boldsymbol{x}, t+T)$.

For our eddy current problem, we are actually looking for a periodic steady state solution as defined in Definition 15.

Let the right hand side be given as $\boldsymbol{f}(\boldsymbol{x}, t)=\hat{\boldsymbol{f}}(\boldsymbol{x}) \cdot \cos (\omega t)$, and suppose we knew a periodic solution $\boldsymbol{u}$ with the same period $T=\frac{2 \pi}{\omega}$. Then - assuming that $\boldsymbol{u}$ is sufficiently smooth in the time variable - we could rewrite it as a Fourier series

$$
\boldsymbol{u}(\boldsymbol{x}, t)=\boldsymbol{u}_{0}^{c}+\sum_{k=1}^{\infty} \boldsymbol{u}_{k}^{c} \cdot \cos (k \omega t)+\boldsymbol{u}_{k}^{s} \cdot \sin (k \omega t) .
$$

Hence, the magnetic field $\boldsymbol{H}=\boldsymbol{H}(\operatorname{curl} \boldsymbol{u})$ is periodic as well and can be written in the form

$$
\begin{aligned}
\boldsymbol{H}(\operatorname{curl} \boldsymbol{u})=\boldsymbol{H}_{0}^{c}(\operatorname{curl} \boldsymbol{u})+ & \sum_{k=1}^{\infty} \boldsymbol{H}_{k}^{c}(\operatorname{curl} \boldsymbol{u}) \cdot \cos (k \omega t) \\
& +\boldsymbol{H}_{k}^{s}(\operatorname{curl} \boldsymbol{u}) \cdot \sin (k \omega t) .
\end{aligned}
$$

We consider equation (5) - together with homogeneous Dirichlet boundary con- 
ditions - in its weak formulation, i.e.

$$
\begin{aligned}
& \underbrace{\frac{2}{T} \int_{0}^{T} \int_{\Omega} \boldsymbol{H}(\operatorname{curl} \boldsymbol{u}) \cdot \operatorname{curl} \boldsymbol{v} \mathrm{d} \boldsymbol{x} \mathrm{dt}}_{=:\langle A(\boldsymbol{u}), \boldsymbol{v}\rangle}+\underbrace{\frac{2}{T} \int_{0}^{T} \int_{\Omega} \sigma \frac{\partial \boldsymbol{u}}{\partial t} \cdot \boldsymbol{v} \mathrm{d} \boldsymbol{x} \mathrm{dt}}_{=:\langle M \boldsymbol{u}, \boldsymbol{v}\rangle} \\
& =\underbrace{\frac{2}{T} \int_{0}^{T} \int_{\Omega} \boldsymbol{f} \cdot \boldsymbol{v} \mathrm{d} \boldsymbol{x} \mathrm{dt}}_{=:\langle F, \boldsymbol{v}\rangle}, \quad \forall \boldsymbol{v} .
\end{aligned}
$$

Apparently, using (27) and (28) as ansatz for the solution, the problem consists in calculating the Fourier coefficients $\boldsymbol{u}_{k}^{c}$ and $\boldsymbol{u}_{k}^{s}$. This means that we regard the operators $A$ and $M$ as defined in (29) as operators mapping from $\boldsymbol{H}_{0}(\mathbf{c u r l})^{\mathbb{N}}$ into its dual space.

For an exact definition of the domain of these operators, assume that the whole region $\Omega$ consists of a conducting and a non-conducting part, $\Omega_{1}$ and $\Omega_{2}$ respectively (cf. Fig. 1). Keeping in mind that eddy current problems are uniquely solvable only up to gradient fields, at least in non-conducting regions, we are looking for Fourier coefficients $\boldsymbol{u}_{k}^{c / s}$ in the space

$$
\boldsymbol{V}:=\boldsymbol{H}_{0}(\operatorname{curl}, \Omega) / \boldsymbol{W}\left(\Omega_{2}\right),
$$

where the gradient fields in $\Omega_{2}, \boldsymbol{W}\left(\Omega_{2}\right)$, are meant as in (10). The constant coefficient

$$
\boldsymbol{u}_{0}^{c} \in \boldsymbol{V}_{0}:=\boldsymbol{H}_{0}(\operatorname{curl}, \Omega) / \boldsymbol{W}(\Omega),
$$

should be divergence-free in the whole domain of consideration.

By Lemma 2, we have $\|\cdot\|_{\boldsymbol{L}_{2}} \simeq\|\operatorname{curl} \cdot\|_{\boldsymbol{L}_{2}}$ in the space $\boldsymbol{V}_{0}$. The space $\boldsymbol{V}$ of solenoidal functions in the non-conducting domain is treated in the following lemma:

Lemma 16. Suppose that the domains $\Omega_{1}$ and $\Omega_{2}$ are multiply connected and have Lipschitz boundary. Let $\bar{\Omega}=\bar{\Omega}_{1} \cup \bar{\Omega}_{2}$ with an interface $\Gamma_{I}=\bar{\Omega}_{1} \cap \bar{\Omega}_{2}$ of positive measure. Then in the space $\boldsymbol{V}$ as defined in (30) we have the estimate

$$
\|\boldsymbol{v}\|_{\boldsymbol{L}_{2}(\Omega)}^{2} \leq c \cdot\left(\|\boldsymbol{v}\|_{\boldsymbol{L}_{2}\left(\Omega_{1}\right)}^{2}+\|\operatorname{curl} \boldsymbol{v}\|_{\boldsymbol{L}_{2}(\Omega)}^{2}\right) .
$$

Proof. Let $\boldsymbol{v} \in \boldsymbol{V}$. In a first step we show

$$
\|\boldsymbol{v}\|_{\boldsymbol{L}_{2}\left(\Omega_{2}\right)} \leq c \cdot\left(\|\operatorname{curl} \boldsymbol{v}\|_{\boldsymbol{L}_{2}\left(\Omega_{2}\right)}+\|\boldsymbol{v} \times \boldsymbol{n}\|_{\boldsymbol{H}^{-\frac{1}{2}}\left(\operatorname{div}_{\Gamma_{I}}, \Gamma_{I}\right)}\right) .
$$

We would like to make use of the result on norm equivalence provided by Lemma 2. However, in order to apply this lemma, we need to construct some $\hat{\boldsymbol{v}}$ with $\hat{\boldsymbol{v}} \times \boldsymbol{n}=0$ on $\partial \Omega_{2}$ and $(\hat{\boldsymbol{v}}, \boldsymbol{w})_{\boldsymbol{L}_{2}\left(\Omega_{2}\right)}=0, \forall \boldsymbol{w} \in \boldsymbol{W}\left(\Omega_{2}\right)$. We know that $\boldsymbol{v}$ has zero tangential trace on $\partial \Omega \cap \partial \Omega_{2}$, but not necessarily on $\Gamma_{I}$. 
By the inverse trace theorem (cf. [7]), we have some $\tilde{\boldsymbol{v}} \in \boldsymbol{H}\left(\mathbf{c u r l}, \Omega_{2}\right)$ with $\tilde{\boldsymbol{v}} \times \boldsymbol{n}=\boldsymbol{v} \times \boldsymbol{n}$ on $\partial \Omega_{2}$ such that

$$
\|\tilde{\boldsymbol{v}}\|_{\boldsymbol{H}\left(\mathbf{c u r l}, \Omega_{2}\right)} \leq c\|\boldsymbol{v} \times \boldsymbol{n}\|_{\boldsymbol{H}^{-\frac{1}{2}\left(\operatorname{div}_{\partial \Omega_{2}}, \partial \Omega_{2}\right)}}=c\|\boldsymbol{v} \times \boldsymbol{n}\|_{\boldsymbol{H}^{-\frac{1}{2}\left(\operatorname{div}_{\Gamma_{I}}, \Gamma_{I}\right)}} .
$$

Now, $(\boldsymbol{v}-\tilde{\boldsymbol{v}}) \times \boldsymbol{n}=0$ on $\partial \Omega_{2}$, but we need to ensure solenoidality:

$$
\begin{aligned}
& \hat{\boldsymbol{v}}:=\boldsymbol{v}-\tilde{\boldsymbol{v}}-\boldsymbol{z}, \\
& \text { with } \boldsymbol{z} \in \boldsymbol{W}\left(\Omega_{2}\right):(\boldsymbol{z}, \boldsymbol{w})_{\boldsymbol{L}_{2}\left(\Omega_{2}\right)}=(\boldsymbol{v}-\tilde{\boldsymbol{v}}, \boldsymbol{w})_{\boldsymbol{L}_{2}\left(\Omega_{2}\right)}, \forall \boldsymbol{w} \in \boldsymbol{W}\left(\Omega_{2}\right) .
\end{aligned}
$$

Thus, we have $\|\boldsymbol{z}\|_{\boldsymbol{L}_{2}\left(\Omega_{2}\right)} \leq\|\tilde{\boldsymbol{v}}\|_{\boldsymbol{L}_{2}\left(\Omega_{2}\right)}$ and, since $\hat{\boldsymbol{v}}$ satisfies the prerequisites of Lemma 2,

$$
\begin{aligned}
& \|\boldsymbol{v}\|_{\boldsymbol{L}_{2}\left(\Omega_{2}\right)} \leq\|\boldsymbol{v}-\tilde{\boldsymbol{v}}\|_{0}+\|\tilde{\boldsymbol{v}}\|_{0} \leq\|\hat{\boldsymbol{v}}\|_{0}+\|\boldsymbol{z}\|_{0}+\|\tilde{\boldsymbol{v}}\|_{0} \\
& \leq c \cdot\|\operatorname{curl} \hat{\boldsymbol{v}}\|_{0}+2\|\tilde{\boldsymbol{v}}\|_{0}=c \cdot\|\operatorname{curl}(\boldsymbol{v}-\tilde{\boldsymbol{v}})\|_{0}+2\|\tilde{\boldsymbol{v}}\|_{0} \\
& \leq c \cdot\|\operatorname{curl} \boldsymbol{v}\|_{0}+c \cdot\|\operatorname{curl} \tilde{\boldsymbol{v}}\|_{0}+2\|\tilde{\boldsymbol{v}}\|_{0} \\
& \leq c \cdot\left(\|\operatorname{curl} \boldsymbol{v}\|_{\boldsymbol{L}_{2}\left(\Omega_{2}\right)}+\|\boldsymbol{v} \times \boldsymbol{n}\|_{\boldsymbol{H}^{-\frac{1}{2}}\left(\operatorname{div}_{\Gamma_{I}}, \Gamma_{I}\right)}\right),
\end{aligned}
$$

where $\|\cdot\|_{0}=\|\cdot\|_{\boldsymbol{L}_{2}\left(\Omega_{2}\right)}$.

The next step yields the desired result, where we take advantage of the bounded trace operator [7]:

$$
\begin{aligned}
\|\boldsymbol{v}\|_{\boldsymbol{L}_{2}(\Omega)}^{2} & =\|\boldsymbol{v}\|_{\boldsymbol{L}_{2}\left(\Omega_{1}\right)}^{2}+\|\boldsymbol{v}\|_{\boldsymbol{L}_{2}\left(\Omega_{2}\right)}^{2} \\
& \leq\|\boldsymbol{v}\|_{\boldsymbol{L}_{2}\left(\Omega_{1}\right)}^{2}+c \cdot\left(\|\operatorname{curl} \boldsymbol{v}\|_{\boldsymbol{L}_{2}\left(\Omega_{2}\right)}^{2}+\|\boldsymbol{v} \times \boldsymbol{n}\|_{\boldsymbol{H}^{-\frac{1}{2}}\left(\operatorname{div}_{\Gamma_{I}}, \Gamma_{I}\right)}^{2}\right) \\
& \leq c \cdot\left(\|\boldsymbol{v}\|_{\boldsymbol{L}_{2}\left(\Omega_{1}\right)}^{2}+\|\operatorname{curl} \boldsymbol{v}\|_{\boldsymbol{L}_{2}\left(\Omega_{2}\right)}^{2}+\|\boldsymbol{v}\|_{\boldsymbol{H}\left(\operatorname{curl}, \Omega_{1}\right)}^{2}\right) \\
& \leq c \cdot\left(\|\boldsymbol{v}\|_{\boldsymbol{L}_{2}\left(\Omega_{1}\right)}^{2}+\|\operatorname{curl} \boldsymbol{v}\|_{\boldsymbol{L}_{2}(\Omega)}^{2}\right) .
\end{aligned}
$$

Finally, we define the space for the Fourier coefficients $\boldsymbol{u}_{k}^{c / s}$ :

$$
\mathbb{V}:=\left\{\boldsymbol{v} \in \boldsymbol{V}_{0} \times\left(\boldsymbol{V}^{2}\right)^{\mathbb{N}}:\|\boldsymbol{v}\|_{\mathbb{V}}<\infty\right\},
$$

with the norm

$$
\begin{aligned}
\|\boldsymbol{v}\|_{\mathbb{V}}^{2} & :=|\boldsymbol{v}|_{M}^{2}+|\boldsymbol{v}|_{A}^{2}, \\
|\boldsymbol{v}|_{M}^{2} & :=\sum_{k=1}^{\infty} k \omega\left(\left\|\boldsymbol{v}_{k}^{c}\right\|_{\boldsymbol{L}_{2}\left(\Omega_{1}\right)}^{2}+\left\|\boldsymbol{v}_{k}^{s}\right\|_{\boldsymbol{L}_{2}\left(\Omega_{1}\right)}^{2}\right), \\
|\boldsymbol{v}|_{A}^{2} & :=\left\|\operatorname{curl} \boldsymbol{v}_{0}^{c}\right\|_{\boldsymbol{L}_{2}(\Omega)}^{2}+\sum_{k=1}^{\infty}\left(\left\|\operatorname{curl} \boldsymbol{v}_{k}^{c}\right\|_{\boldsymbol{L}_{2}(\Omega)}^{2}+\left\|\operatorname{curl} \boldsymbol{v}_{k}^{s}\right\|_{\boldsymbol{L}_{2}(\Omega)}^{2}\right) .
\end{aligned}
$$

We remark that $\|\cdot\|_{\mathbb{V}}$ really is a norm, because for the coefficient $\boldsymbol{v}_{0}^{c}$, which is not covered by the seminorm $|\cdot|_{M}$, we have the norm equivalence with the seminorm 
$\left\|\operatorname{curl} \boldsymbol{v}_{0}^{c}\right\|_{\boldsymbol{L}_{2}}$. Moreover, for the non-conducting domain $\Omega_{2}$, which is not con$\begin{array}{lllll}\text { tained } & \text { in } & \left.\right|_{M} & \text { either, }\end{array}$ Lemma 16 provides the necessary estimate.

By the definition (32), we consider only Fourier series with a solenoidal constant coefficient $\boldsymbol{v}_{0}^{c}$ that are divergence-free in the non-conducting regions. Now we prove that the eddy current problem is uniquely solvable in this space $\mathbb{V}$. Since we can show that the constant coefficient is zero (Theorem 21), this corresponds perfectly well to the results of Section 2, where we showed unique solvability among arbitrary $\boldsymbol{H}$ (curl)-functions that are divergence-free in the non-conducting domain (Corollary 14).

\subsection{Existence and Uniqueness}

With these considerations, we are now able to show the existence of a uniquely defined periodic steady state solution of the eddy current problem (5). Before passing to the main theorem, however, we show some properties of the operators $A$ and $M$ :

Lemma 17. Suppose $\sigma \in L_{\infty}\left(\Omega_{1}\right)$ with $0<\underline{\sigma} \leq \sigma(\boldsymbol{x}) \leq \bar{\sigma}$ almost everywhere. Let the function $s \mapsto \nu(s) \cdot s$ be Lipschitz continuous and strongly monotone, and assume that $\Omega_{1}$ and $\Omega_{2}$ are multiply connected and have Lipschitz boundary.

Then the operators $A: \mathbb{V} \rightarrow \mathbb{V}^{*}$ and $M: \mathbb{V} \rightarrow \mathbb{V}^{*}$ as defined in (29) have the following properties:

$$
\begin{aligned}
\langle A(\boldsymbol{u})-A(\boldsymbol{v}), \boldsymbol{u}-\boldsymbol{v}\rangle & \geq c_{M} \cdot|\boldsymbol{u}-\boldsymbol{v}|_{A}^{2}, \\
\langle A(\boldsymbol{u})-A(\boldsymbol{v}), \boldsymbol{w}\rangle & \leq \frac{3}{2} c_{L} \cdot|\boldsymbol{u}-\boldsymbol{v}|_{A} \cdot|\boldsymbol{w}|_{A}, \\
\langle M \boldsymbol{u}, \boldsymbol{v}\rangle & \leq \bar{\sigma} \cdot|\boldsymbol{u}|_{M} \cdot|\boldsymbol{v}|_{M}, \\
\left\langle M \boldsymbol{u}, \boldsymbol{u}^{\dagger}\right\rangle & \geq \underline{\sigma} \cdot|\boldsymbol{u}|_{M}^{2}, \\
\langle M \boldsymbol{u}, \boldsymbol{u}\rangle & =0
\end{aligned}
$$

where by $\boldsymbol{u}^{\dagger}$ we mean the "rotation" of $\boldsymbol{u}$ in the following sense:

$$
\boldsymbol{u}^{\dagger}=\left(\begin{array}{c}
\boldsymbol{u}^{c} \\
\boldsymbol{u}^{s}
\end{array}\right)^{\dagger}=\left(\begin{array}{c}
\boldsymbol{u}^{s} \\
-\boldsymbol{u}^{c}
\end{array}\right)=\sum_{k=1}^{\infty} \boldsymbol{u}_{k}^{s} \cos (k \omega t)-\boldsymbol{u}_{k}^{c} \sin (k \omega t) .
$$

The constants $c_{M}$ and $c_{L}$ in (36) and (37) are the monotonicity and Lipschitz constants of the function $s \mapsto \nu(s) \cdot s$, respectively.

Proof. All these statements are based on the orthonormality of the functions $\cos (k \omega t), \sin (k \omega t)$ w.r.t. the scalar product $\frac{2}{T}(\cdot, \cdot)_{L_{2}(0, T)}$.

The proof of the properties (36) and (37) relies on the strong monotonicity and Lipschitz continuity of the $\boldsymbol{B}-\boldsymbol{H}$-curve which imply

$$
\begin{aligned}
{\left[\boldsymbol{H}\left(\boldsymbol{B}_{1}\right)-\boldsymbol{H}\left(\boldsymbol{B}_{2}\right)\right]^{T}\left[\boldsymbol{B}_{1}-\boldsymbol{B}_{2}\right] } & \geq c_{M}\left|\boldsymbol{B}_{1}-\boldsymbol{B}_{2}\right|^{2}, \\
\left|\left[\boldsymbol{H}\left(\boldsymbol{B}_{1}\right)-\boldsymbol{H}\left(\boldsymbol{B}_{2}\right)\right]^{T} \boldsymbol{B}_{3}\right| & \leq 3 c_{L}\left|\boldsymbol{B}_{1}-\boldsymbol{B}_{2}\right|\left|\boldsymbol{B}_{3}\right|,
\end{aligned}
$$


for all inductions $\boldsymbol{B}_{1}, \boldsymbol{B}_{2}, \boldsymbol{B}_{3}$ and for the magnetic field $\boldsymbol{H}(\boldsymbol{B})=\nu(|\boldsymbol{B}|) \cdot \boldsymbol{B}$.

The boundedness of $M(38)$ can easily be seen, and (39) is due to the fact that $M$ acts like a rotation:

$$
\begin{aligned}
\left\langle M \boldsymbol{u}, \boldsymbol{u}^{\dagger}\right\rangle & =\frac{2}{T} \int_{0}^{T} \int_{\Omega} \sigma \frac{\partial \boldsymbol{u}}{\partial t} \boldsymbol{u}^{\dagger} \mathrm{d} \boldsymbol{x} \mathrm{dt} \\
& =\int_{\Omega} \sigma \sum_{k} k \omega\left(\left|\boldsymbol{u}_{k}^{c}\right|^{2}+\left|\boldsymbol{u}_{k}^{s}\right|^{2}\right) \mathrm{d} \boldsymbol{x} \geq \underline{\sigma}|\boldsymbol{u}|_{M}^{2} .
\end{aligned}
$$

The orthogonality (40) is clear because of

$$
\int_{0}^{2 \pi} \frac{\partial}{\partial t}[a \cos (t)+b \sin (t)] \cdot[a \cos (t)+b \sin (t)] \mathrm{dt}=b a-a b=0 .
$$

Lemma 18. Let the assumptions of Lemma 17 be valid. Then the operator $K$ defined by

$$
\langle K(\boldsymbol{u}), \boldsymbol{v}\rangle:=\langle A(\boldsymbol{u}), \boldsymbol{v}\rangle+\langle M \boldsymbol{u}, \boldsymbol{v}\rangle,
$$

satisfies the following inf-sup- and sup-sup-condition:

$$
\begin{aligned}
& \inf _{\boldsymbol{u}, \boldsymbol{v} \in \mathbb{V}, \boldsymbol{u} \neq \boldsymbol{v}} \sup _{\boldsymbol{w} \in \mathbb{V}, \boldsymbol{w} \neq 0} \frac{\langle K(\boldsymbol{u})-K(\boldsymbol{v}), \boldsymbol{w}\rangle}{\|\boldsymbol{u}-\boldsymbol{v}\|_{\mathbb{V}}\|\boldsymbol{w}\|_{\mathbb{V}}} \geq \\
& \geq \min \left\{\frac{3}{2} c_{L}, c_{M}\right\} \cdot \frac{\underline{\sigma}}{3 c_{L}+\underline{\sigma}}=: \beta \\
& \sup _{\boldsymbol{u}, \boldsymbol{v} \in \mathbb{V}, \boldsymbol{u} \neq \boldsymbol{v}} \sup _{\boldsymbol{w} \in \mathbb{V}, \boldsymbol{w} \neq 0} \frac{\langle K(\boldsymbol{u})-K(\boldsymbol{v}), \boldsymbol{w}\rangle}{\|\boldsymbol{u}-\boldsymbol{v}\|_{\mathbb{V}}\|\boldsymbol{w}\|_{\mathbb{V}}} \leq 2 \max \left\{\frac{3}{2} c_{L}, \bar{\sigma}\right\}=: \gamma
\end{aligned}
$$

Proof. For the inf-sup-condition, let $\boldsymbol{u}$ and $\boldsymbol{v}$ be given and choose $\boldsymbol{w}=\alpha_{1}(\boldsymbol{u}-$ $\boldsymbol{v})+\alpha_{2}(\boldsymbol{u}-\boldsymbol{v})^{\dagger}$. We distinguish two cases:

- $\frac{3}{2} c_{L}|\boldsymbol{u}-\boldsymbol{v}|_{A}^{2} \leq \frac{1}{2} \sigma|\boldsymbol{u}-\boldsymbol{v}|_{M}^{2}$ : Choose $\alpha_{1}=0, \alpha_{2}=1$. Then by (37) and (39) we have

$$
\begin{aligned}
\frac{\langle K(\boldsymbol{u})-K(\boldsymbol{v}), \boldsymbol{w}\rangle}{\|\boldsymbol{u}-\boldsymbol{v}\|\|\boldsymbol{w}\|}= & \frac{\left\langle A(\boldsymbol{u})-A(\boldsymbol{v}),(\boldsymbol{u}-\boldsymbol{v})^{\dagger}\right\rangle}{\|\boldsymbol{u}-\boldsymbol{v}\|^{2}}+ \\
& +\frac{\left\langle M(\boldsymbol{u}-\boldsymbol{v}),(\boldsymbol{u}-\boldsymbol{v})^{\dagger}\right\rangle}{\|\boldsymbol{u}-\boldsymbol{v}\|^{2}} \\
\geq & \frac{-\left(\frac{3}{2} c_{L}|\boldsymbol{u}-\boldsymbol{v}|_{A}^{2}\right)+\underline{\sigma}|\boldsymbol{u}-\boldsymbol{v}|_{M}^{2}}{|\boldsymbol{u}-\boldsymbol{v}|_{M}^{2}+|\boldsymbol{u}-\boldsymbol{v}|_{A}^{2}} \\
\geq & \frac{\frac{1}{2} \underline{\sigma}|\boldsymbol{u}-\boldsymbol{v}|_{M}^{2}}{\left(1+\frac{\underline{\sigma}}{3 c_{L}}\right)|\boldsymbol{u}-\boldsymbol{v}|_{M}^{2}}=\frac{3}{2} c_{L} \cdot \frac{\underline{\sigma}}{3 c_{L}+\underline{\sigma}}
\end{aligned}
$$


- $\frac{3}{2} c_{L}|\boldsymbol{u}-\boldsymbol{v}|_{A}^{2}>\frac{1}{2} \underline{\sigma}|\boldsymbol{u}-\boldsymbol{v}|_{M}^{2}$ : Choose $\alpha_{1}=1, \alpha_{2}=0$. By (36) and (40) we get

$$
\begin{aligned}
\frac{\langle K(\boldsymbol{u})-K(\boldsymbol{v}), \boldsymbol{w}\rangle}{\|\boldsymbol{u}-\boldsymbol{v}\|\|\boldsymbol{w}\|} & =\frac{\langle A(\boldsymbol{u})-A(\boldsymbol{v}), \boldsymbol{u}-\boldsymbol{v}\rangle+\langle M(\boldsymbol{u}-\boldsymbol{v}), \boldsymbol{u}-\boldsymbol{v}\rangle}{\|\boldsymbol{u}-\boldsymbol{v}\|^{2}} \\
& \geq \frac{c_{M}|\boldsymbol{u}-\boldsymbol{v}|_{A}^{2}+0}{\left(\frac{3 c_{L}}{\underline{\underline{g}}}+1\right)|\boldsymbol{u}-\boldsymbol{v}|_{A}^{2}}=c_{M} \cdot \frac{\underline{\sigma}}{3 c_{L}+\underline{\sigma}}
\end{aligned}
$$

The Lipschitz continuity of $K$, i.e. property (43), follows immediately from the boundedness of $M(38)$, the Lipschitz continuity of $A(37)$ and from $(a+b)^{2} \leq$ $2\left(a^{2}+b^{2}\right), \forall a, b$.

This allows us to prove existence and uniqueness of a periodic steady state solution:

Lemma 19. Let the assumptions of Lemma 17 be valid, suppose moreover that $\nu \in L_{\infty}$ is bounded away from zero: $\nu(s) \geq \underline{\nu}>0$ almost everywhere. Define the space of divergence-free functions

$$
\tilde{\mathbb{V}}:=\left\{\boldsymbol{v} \in \boldsymbol{V}_{0} \times\left(\boldsymbol{V}_{0}^{2}\right)^{\mathbb{N}}:\|\boldsymbol{v}\|_{\mathbb{V}}<\infty\right\} .
$$

Then for $F \in \tilde{\mathbb{V}}^{*}$ the problem

$$
\text { Find } \boldsymbol{u} \in \tilde{\mathbb{V}}: \quad\langle K(\boldsymbol{u}), \boldsymbol{v}\rangle=\langle F, \boldsymbol{v}\rangle, \quad \forall \boldsymbol{v} \in \tilde{\mathbb{V}}
$$

with $K=A+M$ as in (29) or (41), respectively, is uniquely solvable, i.e. there exists a unique divergence-free periodic steady state solution of (5).

Proof. By Browder's and Minty's Theorem it suffices to show that $K$ is strictly monotone, coercive and hemicontinuous. By (36) and (40), we conclude

$$
\begin{aligned}
\langle K(\boldsymbol{u})-K(\boldsymbol{v}), \boldsymbol{u}-\boldsymbol{v}\rangle & =\langle A(\boldsymbol{u})-A(\boldsymbol{v}), \boldsymbol{u}-\boldsymbol{v}\rangle+\langle M(\boldsymbol{u}-\boldsymbol{v}), \boldsymbol{u}-\boldsymbol{v}\rangle \\
& \geq c_{M} \cdot|\boldsymbol{u}-\boldsymbol{v}|_{A}^{2} .
\end{aligned}
$$

In $\tilde{\mathbb{V}}$ we have the norm equivalence according to Lemma 2 , so there $|\cdot|_{A}$ is a norm. Thus strict monotonicity is proved, since $\langle K(\boldsymbol{u})-K(\boldsymbol{v}), \boldsymbol{u}-\boldsymbol{v}\rangle>0$ for $\boldsymbol{u} \neq \boldsymbol{v}$.

Coerciveness follows immediately by

$$
\langle K(\boldsymbol{u}), \boldsymbol{u}\rangle \geq \underline{\nu} \cdot|\boldsymbol{u}|_{A}^{2}
$$

and again the norm equivalence, and hemicontinuity is obvious.

Theorem 20. Let the assumptions of Lemma 17 be valid, suppose moreover $\nu \geq 0$ almost everywhere.

Then for each $F \in \mathbb{V}^{*}$ that is divergence-free in the conducting region, the problem

$$
\text { Find } \boldsymbol{u} \in \mathbb{V}: \quad\langle K(\boldsymbol{u}), \boldsymbol{v}\rangle=\langle F, \boldsymbol{v}\rangle, \quad \forall \boldsymbol{v} \in \mathbb{V},
$$

is uniquely solvable. 
Proof. For showing unique solvability, we proceed as follows: According to Lemma 19 we observe solvability in the subspace of divergence-free functions. ${ }^{1}$ So first we show that the problem is solvable even in the larger space $\mathbb{V}$, and finally we conclude uniqueness by the inf-sup-condition (42).

By Lemma 19, we know

$$
\exists \boldsymbol{u} \in \tilde{\mathbb{V}} \subset \mathbb{V}: \quad\langle K(\boldsymbol{u}), \boldsymbol{v}\rangle=\langle F, \boldsymbol{v}\rangle, \quad \forall \boldsymbol{v} \in \tilde{\mathbb{V}} .
$$

Suppose now that we face a test function $\boldsymbol{w} \in \mathbb{V}, \boldsymbol{w}=\boldsymbol{v}_{1}+\boldsymbol{v}_{2}$ with $\boldsymbol{v}_{1} \in \tilde{\mathbb{V}}$ and $\boldsymbol{v}_{2} \in \boldsymbol{W}\left(\Omega_{1}\right)$, i.e. a gradient field in the conducting region. Then the left hand side of equation (46) remains unchanged, because

$$
\left\langle K(\boldsymbol{u}), \boldsymbol{v}_{2}\right\rangle=\left\langle A(\boldsymbol{u}), \boldsymbol{v}_{2}\right\rangle+\left\langle M \boldsymbol{u}, \boldsymbol{v}_{2}\right\rangle=0+0 .
$$

Here, we take advantage of the solenoidality of $\boldsymbol{u}$, which implies $\left\langle M \boldsymbol{u}, \boldsymbol{v}_{2}\right\rangle=0$, and of the fact that the curl of a gradient field vanishes. Since $F$ is divergencefree in the conducting region, we have $\left\langle F, \boldsymbol{v}_{2}\right\rangle=0$, i.e. the right hand side of (46) stays the same as well.

Thus we have proved the solvability of (45). Remains to show the uniqueness: Suppose we have two solutions $\boldsymbol{u}_{1}$ and $\boldsymbol{u}_{2}$. Then we know

$$
\left\langle K\left(\boldsymbol{u}_{1}\right)-K\left(\boldsymbol{u}_{2}\right), \boldsymbol{v}\right\rangle=0, \quad \forall \boldsymbol{v} \in \mathbb{V} .
$$

On the other hand, the inf-sup-condition (42) ensures

$$
\beta\left\|\boldsymbol{u}_{1}-\boldsymbol{u}_{2}\right\| \leq \sup _{\boldsymbol{v} \in \mathbb{V},\|\boldsymbol{v}\|=1}\left\langle K\left(\boldsymbol{u}_{1}\right)-K\left(\boldsymbol{u}_{2}\right), \boldsymbol{v}\right\rangle=0
$$

thus $\boldsymbol{u}_{1}=\boldsymbol{u}_{2}$.

\subsection{Reduction to Odd Harmonics}

Theorem 20 states that for given divergence-free current source $\boldsymbol{f}$, there is exactly one periodic steady state solution $\boldsymbol{u} \in \mathbb{V}$. In the following, we use this result to show that we do not require the even coefficients in the Fourier series for $\boldsymbol{u}$.

In order to keep notation simple, we use $\boldsymbol{u}$ to denote the sequence of Fourier coefficients and $\boldsymbol{u}(t)$ to signify the periodic function that is determined by these coefficients according to (27), and similarly for $\boldsymbol{f}$.

Since odd modes $\cos ((2 k+1) \omega t), \sin ((2 k+1) \omega t), k \in \mathbb{N}$, change the sign when shifted by half a period, the condition

$$
\boldsymbol{v}\left(t+\frac{\pi}{\omega}\right)=-\boldsymbol{v}(t), \quad \forall t
$$

is an equivalent characterization of the property $\boldsymbol{v}_{2 k}^{c}=\boldsymbol{v}_{2 k}^{s}=0, \forall k \in \mathbb{N}_{0}$, for any function $\boldsymbol{v}=\boldsymbol{v}_{0}^{c}+\sum \boldsymbol{v}_{k}^{c} \cos (k \omega t)+\boldsymbol{v}_{k}^{s} \sin (k \omega t)$. The current source $\boldsymbol{f}=\hat{\boldsymbol{f}} \cdot \cos (\omega t)$ apparently satisfies $(47)$.

\footnotetext{
${ }^{1}$ We remark that $\nu \geq 0$ is sufficient for monotonicity, what ensures solvability already.
} 
Due to the unique solvability of (5), it is fairly easy to see that $\boldsymbol{f}$ satisfying (47) implies the same property for the periodic steady state solution:

For a given right hand side $\boldsymbol{f}(\cdot)$, we get the unique solution $\boldsymbol{u}$. Shifting the right hand side to $\tilde{\boldsymbol{f}}(\cdot)=\boldsymbol{f}\left(\cdot+\frac{\pi}{\omega}\right)$, we obtain the solution $\tilde{\boldsymbol{u}}$. On the other hand, $\bar{f}=-\boldsymbol{f}$ leads to the result $\overline{\boldsymbol{u}}$. Note that, since $\boldsymbol{f}\left(\cdot+\frac{\pi}{\omega}\right)=-\boldsymbol{f}(\cdot)$, we have $\tilde{\boldsymbol{u}}=\overline{\boldsymbol{u}}$.

Clearly, $\boldsymbol{u}\left(\cdot+\frac{\pi}{\omega}\right)$ is a periodic steady state solution of (5) with right hand side $\tilde{\boldsymbol{f}}$. Because of Theorem 20, we have $\tilde{\boldsymbol{u}}(\cdot)=\boldsymbol{u}\left(\cdot+\frac{\pi}{\omega}\right)$.

On the other hand, $-\boldsymbol{u}$ apparently solves (5) with right hand side $\bar{f}$ and so $-\boldsymbol{u}=\overline{\boldsymbol{u}}$ because of the uniqueness.

So altogether we have the relation

$$
\boldsymbol{u}\left(\cdot+\frac{\pi}{\omega}\right)=\tilde{\boldsymbol{u}}(\cdot)=\overline{\boldsymbol{u}}(\cdot)=-\boldsymbol{u}(\cdot),
$$

i.e. $\boldsymbol{u}$ satisfies (47) and can thus be described by odd harmonics, what proves the following theorem:

Theorem 21. Let the current source $\boldsymbol{f}$ satisfy (47). Then the unique periodic steady state solution of (5) with right hand side $\boldsymbol{f}$ satisfies (47) as well and can accordingly be entirely represented by odd harmonics, i.e.

$$
\boldsymbol{u}(\boldsymbol{x}, t)=\sum_{k=0}^{\infty} \boldsymbol{u}_{2 k+1}^{c}(\boldsymbol{x}) \cdot \cos ((2 k+1) \omega t)+\boldsymbol{u}_{2 k+1}^{s}(\boldsymbol{x}) \cdot \sin ((2 k+1) \omega t) .
$$

Remark 22. Since the solution $\boldsymbol{u}(\boldsymbol{x}, t)$ depends only on odd harmonics, the magnetic field $\boldsymbol{H}(\boldsymbol{\operatorname { c u r l }} \boldsymbol{u})$ has the same property:

$$
\begin{aligned}
\boldsymbol{H}\left(t+\frac{\pi}{\omega}\right) & =\nu\left(\left|\operatorname{curl} \boldsymbol{u}\left(t+\frac{\pi}{\omega}\right)\right|\right) \cdot \operatorname{curl} \boldsymbol{u}\left(t+\frac{\pi}{\omega}\right)= \\
& =\nu(|-\operatorname{curl} \boldsymbol{u}(t)|) \cdot(-\operatorname{curl} \boldsymbol{u}(t))=-\boldsymbol{H}(t) .
\end{aligned}
$$

\subsection{Time Discretization by means of a Multiharmonic Ansatz}

For numerical calculations, we cannot use the whole Fourier series, but only a finite sum

$$
\boldsymbol{u}(\boldsymbol{x}, t) \sim \sum_{k=0}^{N}\left[\boldsymbol{u}_{k}^{c}(\boldsymbol{x}) \cdot \cos (k \omega t)+\boldsymbol{u}_{k}^{s}(\boldsymbol{x}) \cdot \sin (k \omega t)\right] .
$$

We use this so-called multiharmonic ansatz for the current source $f$ and for the magnetic field $\boldsymbol{H}(\operatorname{curl} \boldsymbol{u})$ as well, i.e. we truncate the Fourier series expansion 
at the $N$-th coefficient. Consequently, the problem that we deal with reads

$$
\begin{aligned}
\operatorname{curl} & \sum_{k=0}^{N}\left[\boldsymbol{H}_{k}^{c}(\operatorname{curl} \boldsymbol{u}) \cdot \cos (k \omega t)+\boldsymbol{H}_{k}^{s}(\operatorname{curl} \boldsymbol{u}) \cdot \sin (k \omega t)\right]+ \\
+ & \omega \sigma \sum_{k=0}^{N} k\left[\boldsymbol{u}_{k}^{s} \cdot \cos (k \omega t)-\boldsymbol{u}_{k}^{c} \cdot \sin (k \omega t)\right] \\
= & \sum_{k=0}^{N}\left[\boldsymbol{f}_{k}^{c} \cdot \cos (k \omega t)+\boldsymbol{f}_{k}^{s} \cdot \sin (k \omega t)\right]
\end{aligned}
$$

We test this equation with $\cos (m \omega t)$ and $\sin (m \omega t)$ and integrate by $t$, taking advantage of the orthogonality

$$
\begin{aligned}
& \frac{\omega}{\pi} \int_{0}^{\frac{2 \pi}{\omega}} \cos (k \omega t) \cos (m \omega t) d t=\delta_{k m} \\
& \frac{\omega}{\pi} \int_{0}^{\frac{2 \pi}{\omega}} \cos (k \omega t) \sin (m \omega t) d t=0 \\
& \frac{\omega}{\pi} \int_{0}^{\frac{2 \pi}{\omega}} \sin (k \omega t) \sin (m \omega t) d t=\delta_{k m} .
\end{aligned}
$$

Together with the fact that all even harmonics are zero (Theorem 21), this leads to the following system of equations in space:

$$
\begin{aligned}
& \frac{\omega}{\pi} \int_{0}^{\frac{2 \pi}{\omega}}(49) \cdot\left\{\begin{array}{l}
\cos (m \omega t) \\
\sin (m \omega t)
\end{array}\right\} d t \Longrightarrow \\
& \operatorname{curl}\left(\begin{array}{c}
\boldsymbol{H}_{1}^{c}(\operatorname{curl} \boldsymbol{u}) \\
\boldsymbol{H}_{1}^{s}(\operatorname{curl} \boldsymbol{u}) \\
\vdots \\
\boldsymbol{H}_{2 n+1}^{c}(\operatorname{curl} \boldsymbol{u}) \\
\boldsymbol{H}_{2 n+1}^{s}(\operatorname{curl} \boldsymbol{u})
\end{array}\right)+ \\
& +\omega \sigma \underbrace{\left(\begin{array}{ccccc}
0 & 1 & & & \\
-1 & 0 & & & \\
& & \ddots & & \\
& & & 0 & 2 n+1
\end{array}\right)}_{=: D}\left(\begin{array}{c}
\boldsymbol{u}_{1}^{c} \\
\boldsymbol{u}_{1}^{s} \\
\vdots \\
\boldsymbol{u}_{2 n+1}^{c} \\
\boldsymbol{u}_{2 n+1}^{s}
\end{array}\right)=\left(\begin{array}{c}
\boldsymbol{f}_{1}^{c} \\
\boldsymbol{f}_{1}^{s} \\
\vdots \\
\boldsymbol{f}_{2 n+1}^{c} \\
\boldsymbol{f}_{2 n+1}^{s}
\end{array}\right) \text {, }
\end{aligned}
$$

where we assume $N=2 n+1$.

For the sake of better readability, we introduce the abbreviation $\boldsymbol{H}=\left(\boldsymbol{H}_{1}^{c}, \boldsymbol{H}_{1}^{s}\right.$, $\left.\ldots, \boldsymbol{H}_{2 n+1}^{s}\right)^{T}$ for the Fourier coefficients of the magnetic field, and analogously we write $\boldsymbol{u}$ for $\left(\boldsymbol{u}_{1}^{c}, \ldots, \boldsymbol{u}_{2 n+1}^{s}\right)^{T}$ and $\boldsymbol{f}$ for $\left(\boldsymbol{f}_{1}^{c}, \ldots, \boldsymbol{f}_{2 n+1}^{s}\right)^{T}$. Now the problem that we have to solve, i.e. (50) together with homogeneous Dirichlet boundary 
conditions, can be written in a more compact way:

$$
\begin{aligned}
\operatorname{curl} \boldsymbol{H}(\operatorname{curl} \boldsymbol{u})+\omega \sigma \boldsymbol{D} \boldsymbol{u} & =\boldsymbol{f}, & & \text { in } \Omega, \\
\boldsymbol{u} \times \boldsymbol{n} & =0, & & \text { on } \Gamma,
\end{aligned}
$$

with the matrix $\boldsymbol{D}$ from (50).

\subsection{The Error due to Truncation of the Fourier Series}

In this section we analyze the error that we have introduced by the multiharmonic ansatz, i.e. by truncating the Fourier series at some finite index $N$.

The analysis splits in three parts: First, we claim that the discretization error can be estimated by the best approximation error, analogously to Cea's Lemma (e.g. [5]) for linear elliptic problems or rather to Babuška's and Aziz' lemma for mixed problems [2]. Secondly, we further estimate the approximation error by analyzing the convergence of Fourier series for sufficiently smooth functions. Finally, we quote a regularity result which shows that the solution is adequately smooth.

Recall that the original problem reads as:

$$
\text { Find } \boldsymbol{u} \in \mathbb{V}: \quad\langle K(\boldsymbol{u}), \boldsymbol{v}\rangle=\langle F, \boldsymbol{v}\rangle, \quad \forall \boldsymbol{v} \in \mathbb{V},
$$

with the operator $K: \mathbb{V} \rightarrow \mathbb{V}^{*}, K=A+M$ as in (29) or (41), respectively. The discrete problem, which has been stated in (49), can be written in abstract form as

$$
\text { Find } \boldsymbol{u}_{N} \in \mathbb{V}_{N}: \quad\left\langle K\left(\boldsymbol{u}_{N}\right), \boldsymbol{v}_{N}\right\rangle=\left\langle F, \boldsymbol{v}_{N}\right\rangle, \quad \forall \boldsymbol{v}_{N} \in \mathbb{V}_{N},
$$

where the discrete space $\mathbb{V}_{N}$ corresponds to finite sums of Fourier coefficients:

$$
\mathbb{V}_{N}:=\boldsymbol{V}_{0} \times\left(\boldsymbol{V}^{2}\right)^{N}
$$

The space $\mathbb{V}_{N} \subset \mathbb{V}$ should be equipped with the same norm $\|\cdot\|_{\mathbb{V}}$.

We remark that - due to Theorem 21 - in practice we consider only the odd harmonics in $\mathbb{V}_{N}$. Moreover we emphasize that the operator $K$ in the discrete problem is actually the same as in the original problem where we consider the complete Fourier series. This seems surprising at first glance, since for $\boldsymbol{u} \in \mathbb{V}_{N}$, in general $\boldsymbol{H}(\operatorname{curl} \boldsymbol{u}) \notin \mathbb{V}_{N}$, but only $\boldsymbol{H}(\operatorname{curl} \boldsymbol{u}) \in \mathbb{V}$. However, the Fourier coefficients $\boldsymbol{H}_{k}$ of the magnetic field for $k>N$ do not appear in the equation since we test only with functions $\boldsymbol{v} \in \mathbb{V}_{N}$.

Thus we have the following result:

Lemma 23. Suppose the assumptions of Lemma 17 are valid and that the right hand side is divergence-free in $\Omega_{1}$. Denote the solutions of (45) and (52) by $\boldsymbol{u}$ and $\boldsymbol{u}_{N}$, respectively.

Then we have

$$
\left\|\boldsymbol{u}-\boldsymbol{u}_{N}\right\|_{\mathbb{V}} \leq c \inf _{\boldsymbol{v}_{N} \in \mathbb{V}_{N}}\left\|\boldsymbol{u}-\boldsymbol{v}_{N}\right\|_{\mathbb{V}}
$$


Proof. The proof exploits the Galerkin orthogonality and the fact that the infsup-condition (42) holds for the discrete problem as well: As can be seen in the proof of Lemma 18, for $\boldsymbol{u}_{N}, \boldsymbol{v}_{N} \in \mathbb{V}_{N}$ we have

$$
\sup _{\boldsymbol{w}_{N} \in \mathbb{V}_{N}, \boldsymbol{w}_{N} \neq 0} \frac{\left\langle K\left(\boldsymbol{u}_{N}\right)-K\left(\boldsymbol{v}_{N}\right), \boldsymbol{w}_{N}\right\rangle}{\left\|\boldsymbol{w}_{N}\right\|_{\mathbb{V}}} \geq \beta\left\|\boldsymbol{u}_{N}-\boldsymbol{v}_{N}\right\|_{\mathbb{V}}
$$

Consequently, for arbitrary $\boldsymbol{v}_{N} \in \mathbb{V}_{N}$ we can estimate the discretization error in the following way:

$$
\begin{aligned}
\left\|\boldsymbol{u}-\boldsymbol{u}_{N}\right\| & \leq\left\|\boldsymbol{u}-\boldsymbol{v}_{N}\right\|+\left\|\boldsymbol{u}_{N}-\boldsymbol{v}_{N}\right\| \\
& \leq\left\|\boldsymbol{u}-\boldsymbol{v}_{N}\right\|+\frac{1}{\beta} \sup _{\substack{\boldsymbol{w}_{N} \in \mathbb{V}_{N}, \boldsymbol{w}_{N} \neq 0}} \frac{\left\langle K\left(\boldsymbol{u}_{N}\right)-K\left(\boldsymbol{v}_{N}\right), \boldsymbol{w}_{N}\right\rangle}{\left\|\boldsymbol{w}_{N}\right\|} \\
& \leq\left\|\boldsymbol{u}-\boldsymbol{v}_{N}\right\|+\frac{1}{\beta} \underbrace{\sup _{=0} \frac{\left\langle K\left(\boldsymbol{u}_{N}\right)-K(\boldsymbol{u}), \boldsymbol{w}_{N}\right\rangle}{\left\|\boldsymbol{w}_{N}\right\|}}_{\substack{\boldsymbol{w}_{N} \in \mathbb{V}_{N}, \boldsymbol{w}_{N} \neq 0}} \\
& +\frac{1}{\beta} \sup _{\substack{\boldsymbol{w}_{N} \in \mathbb{V}_{N}, \boldsymbol{w}_{N} \neq 0}} \frac{\left\langle K(\boldsymbol{u})-K\left(\boldsymbol{v}_{N}\right), \boldsymbol{w}_{N}\right\rangle}{\left\|\boldsymbol{w}_{N}\right\|} \\
& \leq\left\|\boldsymbol{u}-\boldsymbol{v}_{N}\right\|+\frac{1}{\beta} \gamma\left\|\boldsymbol{u}-\boldsymbol{v}_{N}\right\| .
\end{aligned}
$$

Obviously, the best approximation error to some function $\boldsymbol{u}=\boldsymbol{u}_{0}^{c}+\sum_{k=1}^{\infty} \boldsymbol{u}_{k}^{c} \cos (k \omega t)+$ $\boldsymbol{u}_{k}^{s} \sin (k \omega t)$ can be estimated by

$$
\inf _{\boldsymbol{v}_{N} \in \mathbb{V}_{N}}\left\|\boldsymbol{u}-\boldsymbol{v}_{N}\right\|_{\mathbb{V}} \leq\left\|\boldsymbol{u}-\tilde{\boldsymbol{u}}_{N}\right\|_{\mathbb{V}}
$$

where $\tilde{\boldsymbol{u}}_{N}=\boldsymbol{u}_{0}^{c}+\sum_{k=1}^{N} \boldsymbol{u}_{k}^{c} \cos (k \omega t)+\boldsymbol{u}_{k}^{s} \sin (k \omega t)$ is the truncated Fourier series. $^{2}$

This difference between the partial sum and the whole Fourier series converges to zero for $N \rightarrow \infty$, provided the function $\boldsymbol{u}$ is smooth enough. More precisely, we have the following estimate:

Lemma 24. If $\boldsymbol{u}(\boldsymbol{x}, t) \in H^{1}((0, T), \boldsymbol{H}(\mathbf{c u r l})) \cap H^{2}\left((0, T), \boldsymbol{L}_{2}\right)$, then $\boldsymbol{u}$ can be represented by means of a Fourier series. Moreover, the error between the function $\boldsymbol{u}(\boldsymbol{x}, t)$ and the truncated Fourier series $\tilde{\boldsymbol{u}}_{N}=\boldsymbol{u}_{0}^{c}+\sum_{k=1}^{N} \boldsymbol{u}_{k}^{c} \cos (k \omega t)+$ $\boldsymbol{u}_{k}^{s} \sin (k \omega t)$ can be estimated by

$$
\begin{aligned}
& \left\|\boldsymbol{u}-\tilde{\boldsymbol{u}}_{N}\right\|_{L_{2}((0, T), \boldsymbol{H}(\operatorname{curl})) \cap H^{1}\left((0, T), \boldsymbol{L}_{2}\right)} \leq \\
& \quad \leq c N^{-1}\|\boldsymbol{u}\|_{H^{1}((0, T), \boldsymbol{H}(\operatorname{curl})) \cap H^{2}\left((0, T), \boldsymbol{L}_{2}\right)},
\end{aligned}
$$

${ }^{2}$ Note that for $\boldsymbol{u}$ solving (45), $\tilde{\boldsymbol{u}}_{N}$ does not necessarily solve (52). 
provided the coefficients are calculated in the usual way:

$$
\boldsymbol{u}_{k}^{c}=\frac{2}{T} \int_{0}^{T} \boldsymbol{u}(\boldsymbol{x}, t) \cos (k \omega t) \mathrm{dt}, \text { and } \boldsymbol{u}_{k}^{s}=\frac{2}{T} \int_{0}^{T} \boldsymbol{u}(\boldsymbol{x}, t) \sin (k \omega t) \mathrm{dt} .
$$

Proof. Since $\boldsymbol{u}$ is continuous in the time variable, its Fourier series expansion converges. Remains to analyze the rate of convergence. Therefore, we take a close look at the norm in the space $\mathbb{X}_{m}:=H^{m}((0, T), \boldsymbol{H}($ curl $)) \cap$ $H^{m+1}\left((0, T), \boldsymbol{L}_{2}\right)$ for $m \in \mathbb{N}_{0}$ and the way it is calculated for a Fourier series. For each $\boldsymbol{v} \in \mathbb{X}_{m}$, we have the representation

$$
\begin{aligned}
\|\boldsymbol{v}\|_{\mathbb{X}_{m}}^{2}=\int_{0}^{T}\left[|\boldsymbol{v}(t)|_{1}^{2}+\left|\boldsymbol{v}^{\prime}(t)\right|_{1}^{2}+\ldots+\left|\boldsymbol{v}^{(m)}(t)\right|_{1}^{2}+\right. \\
\left.\quad+\|\boldsymbol{v}(t)\|_{0}^{2}+\left\|\boldsymbol{v}^{\prime}(t)\right\|_{0}^{2}+\ldots+\left\|\boldsymbol{v}^{(m+1)}\right\|_{0}^{2}\right] \mathrm{dt} \\
=\frac{T}{2} \sum_{k=0}^{\infty}\left[\left[1+(k \omega)^{2}+\ldots+(k \omega)^{2 m}\right]\left(\left|\boldsymbol{v}_{k}^{c}\right|_{1}^{2}+\left|\boldsymbol{v}_{k}^{s}\right|_{1}^{2}\right)+\right. \\
\left.\quad+\left[1+\ldots+(k \omega)^{2(m+1)}\right]\left(\left\|\boldsymbol{v}_{k}^{c}\right\|_{0}^{2}+\left\|\boldsymbol{v}_{k}^{s}\right\|_{0}^{2}\right)\right] .
\end{aligned}
$$

Here and in the following, we mean by $\|\cdot\|_{0}$ the $\boldsymbol{L}_{2}$-norm, and by $|\cdot|_{1}$ the $\boldsymbol{H}$ (curl)-seminorm.

Consequently, the convergence speed can be estimated by

$$
\begin{aligned}
&\left\|\boldsymbol{u}-\tilde{\boldsymbol{u}}_{N}\right\|_{\mathbb{X}_{0}}^{2}= \frac{T}{2} \sum_{k=N+1}^{\infty}\left[\left(\left|\boldsymbol{u}_{k}^{c}\right|_{1}^{2}+\left|\boldsymbol{u}_{k}^{s}\right|_{1}^{2}\right)+\right. \\
&\left.+\left[1+(k \omega)^{2}\right]\left(\left\|\boldsymbol{u}_{k}^{c}\right\|_{0}^{2}+\left\|\boldsymbol{u}_{k}^{s}\right\|_{0}^{2}\right)\right] \\
& \leq \frac{T}{2} \max _{k \geq N+1} \frac{1}{1+(k \omega)^{2}} \cdot \\
& \cdot \sum_{k=N+1}^{\infty}\left[\left[1+(k \omega)^{2}\right]\left(\left|\boldsymbol{u}_{k}^{c}\right|_{1}^{2}+\left|\boldsymbol{u}_{k}^{s}\right|_{1}^{2}\right)+\right. \\
& \leq\left.\quad+\left[1+(k \omega)^{2}+(k \omega)^{4}\right]\left(\left\|\boldsymbol{u}_{k}^{c}\right\|_{0}^{2}+\left\|\boldsymbol{u}_{k}^{s}\right\|_{0}^{2}\right)\right] \\
& \frac{T}{2} \frac{1}{1+[(N+1) \omega]^{2}}\|\boldsymbol{u}\|_{\mathbb{X}_{1}}^{2} \leq c N^{-2}\|\boldsymbol{u}\|_{\mathbb{X}_{1}}^{2} .
\end{aligned}
$$

Sufficient regularity of the periodic steady state solution $\boldsymbol{u}(\boldsymbol{x}, t)$ of $(5)$ can be deduced by the regularity of the right hand side $\boldsymbol{f}(\boldsymbol{x}, t)=\hat{\boldsymbol{f}}(\boldsymbol{x}) \cdot \cos (\omega t)$ : Analoguously to $\left[12, \S 7.1 .3\right.$, Theorem 5], we can deduce from $\boldsymbol{f} \in H^{1}\left((0, T), \boldsymbol{L}_{2}(\Omega)\right)$ 
that $\boldsymbol{u} \in H^{1}\left((0, T), \boldsymbol{H}_{0}(\mathbf{c u r l})\right) \cap H^{2}\left((0, T), \boldsymbol{H}_{0}(\mathbf{c u r l})^{*}\right)$. This result can be immediately carried over to the situation of $\boldsymbol{f} \in H^{2}\left((0, T), \boldsymbol{L}_{2}(\Omega)\right)$, what yields even more than the desired regularity $\boldsymbol{u} \in \mathbb{X}_{1}$.

Now, since $\|\cdot\|_{\mathbb{V}} \leq c\|\cdot\|_{\mathbb{X}_{0}}$, the Lemmata 23 and 24 together with the knowledge about regularity can be combined to achieve the final result of this section:

Theorem 25. Assume $\boldsymbol{f}(\boldsymbol{x}, t) \in H^{2}\left((0, T), \boldsymbol{L}_{2}\right), \boldsymbol{f}$ divergence-free in the conducting region, and suppose the assumptions of Lemma 17 are valid. Denote the periodic steady state solution of (5), i.e. the solution of (45), by $\boldsymbol{u}=\boldsymbol{u}(\boldsymbol{x}, t)$, and let $\boldsymbol{u}_{N}$ denominate the solution of the multiharmonic problem (52).

Then we have

$$
\left\|\boldsymbol{u}-\boldsymbol{u}_{N}\right\|_{\mathbb{V}} \leq c N^{-1}\|\boldsymbol{u}\|_{\mathbb{X}_{1}}=c(\boldsymbol{u}) \cdot N^{-1}
$$

\section{Regularization}

As we have seen in Sections 2 and 3, the eddy current problem (5) is uniquely solvable only in the space of divergence-free functions (at least in the nonconducting regions). This circumstance has to be considered in the numerical solution: One can try to tackle the problem in the factor space of solenoidal functions $\boldsymbol{V} / \boldsymbol{W}$ or work with an equivalent mixed formulation.

However, we prefer to follow a different approach: We slightly perturb the problem by introducing a small regularization parameter $\epsilon>0$ in the nonconducting regions, more precisely by replacing the conductivity coefficient $\sigma$ by

$$
\sigma_{\epsilon}(\boldsymbol{x})=\max \{\sigma(\boldsymbol{x}), \epsilon\} .
$$

The resulting perturbed problem then is uniquely solvable in the whole space $\boldsymbol{H}$ (curl).

In the following, we motivate and justify this regularization at the example of the linear harmonic problem. Also, we emphasize that the results achieved in this section can be directly carried over to the nonlinear multiharmonic problem. Supposing that the reluctivity $\nu=\nu(\boldsymbol{x})$ does not depend on the induction $\boldsymbol{B}=\operatorname{curl} \boldsymbol{u}$ and considering only the base harmonic leads to the following variational equation (cf. Section 3):

$$
\begin{array}{r}
\int_{\Omega} \nu \operatorname{curl}\left(\begin{array}{c}
\boldsymbol{v}^{c} \\
\boldsymbol{v}^{s}
\end{array}\right)^{T} \operatorname{curl}\left(\begin{array}{l}
\boldsymbol{u}^{c} \\
\boldsymbol{u}^{s}
\end{array}\right)+\omega \sigma\left(\begin{array}{l}
\boldsymbol{v}^{c} \\
\boldsymbol{v}^{s}
\end{array}\right)^{T}\left(\begin{array}{cc}
0 & 1 \\
-1 & 0
\end{array}\right)\left(\begin{array}{l}
\boldsymbol{u}^{c} \\
\boldsymbol{u}^{s}
\end{array}\right) \mathrm{d} \boldsymbol{x}= \\
=\int_{\Omega}\left(\begin{array}{c}
\boldsymbol{v}^{c} \\
\boldsymbol{v}^{s}
\end{array}\right)^{T}\left(\begin{array}{l}
\boldsymbol{f}^{c} \\
\boldsymbol{f}^{s}
\end{array}\right) \mathrm{d} \boldsymbol{x}, \quad \forall\left(\begin{array}{l}
\boldsymbol{v}^{c} \\
\boldsymbol{v}^{s}
\end{array}\right) \in \boldsymbol{H}_{0}(\mathbf{c u r l})^{2} .
\end{array}
$$

In Theorem 20 we have shown that there exists a unique divergence-free periodic steady state solution of the eddy current problem (5). Since for linear problems and harmonic source current, this solution obviously depends only on the base frequency, equation (57) is uniquely solvable in the space of divergence-free functions. 
Another way to show existence and uniqueness for this linear problem is to employ the knowledge about mixed finite element methods and saddle point problems.

\subsection{Mixed Problems}

Mixed finite element methods are concerned with the solution of problems of the following form:

$$
\begin{aligned}
& \text { Find }(u, \phi) \in V \times W: \\
& \begin{array}{rlrl}
a(u, v)+b(v, \phi) & =\langle F, v\rangle, & & \forall v \in V, \\
b(u, \psi) & =\langle G, \psi\rangle, & \forall \psi \in W .
\end{array}
\end{aligned}
$$

We introduce the space

$$
V_{b}:=\{v \in V: b(v, \psi)=0, \forall \psi \in W\} .
$$

Remark 26. Note that, if a pair $(u, \phi)$ satisfies (58) with $G=0$, then the first argument $u$ is a solution of the variational problem

$$
\text { Find } u \in V_{b}: a(u, v)=\langle F, v\rangle, \quad \forall v \in V_{b} .
$$

We quote a well-known theorem about unique solvability of the mixed problem (58):

Theorem $27([6])$. Let the bilinear form $a(\cdot, \cdot)$ be continuous on $V \times V$ and $V_{b}$-elliptic, i.e.

$$
\exists \alpha>0: a(v, v) \geq \alpha\|v\|_{V}^{2}, \quad \forall v \in V_{b} .
$$

Assume moreover that the bilinear form $b(\cdot, \cdot)$ is continuous on $V \times W$ and that it satisfies the so-called inf-sup-condition

$$
\exists \beta>0: \inf _{\psi \in W, \psi \neq 0} \sup _{v \in V, v \neq 0} \frac{b(v, \psi)}{\|v\|_{V}\|\psi\|_{W}} \geq \beta .
$$

Then, for each $F \in V^{*}$ and $G \in W^{*}$, problem (58) has a unique solution.

We now want to rewrite the variational equation (57) as an equivalent mixed problem. For this task, we define $\boldsymbol{V}:=\boldsymbol{H}_{0}(\mathbf{c u r l})^{2}$ and $\boldsymbol{W}:=\boldsymbol{W}(\Omega)^{2}$. If we define the bilinear form $b(\cdot, \cdot)$ as

$$
b(\boldsymbol{v}, \boldsymbol{\phi}):=\int_{\Omega}\left(\begin{array}{l}
\boldsymbol{v}^{c} \\
\boldsymbol{v}^{s}
\end{array}\right)^{T}\left(\begin{array}{l}
\phi^{c} \\
\boldsymbol{\phi}^{s}
\end{array}\right) \mathrm{d} \boldsymbol{x}, \quad \forall \boldsymbol{v} \in \boldsymbol{V}, \boldsymbol{\phi} \in \boldsymbol{W},
$$

the space $\boldsymbol{V}_{b}$ (59) introduced for the analysis of mixed finite element methods equals exactly the space of all weakly divergence-free functions in $\boldsymbol{V}=$ $\boldsymbol{H}_{0}(\mathbf{c u r l})^{2}$. As we have seen in the discussion about existence and uniqueness in Section 2, this space is essential for eddy current problems, since in nonconducting regions uniqueness can only be guaranteed up to the set of gradient fields. 
Let us now define

$$
k(\boldsymbol{u}, \boldsymbol{v}):=\int_{\Omega}\left[\nu \operatorname{curl}\left(\begin{array}{c}
\boldsymbol{v}^{c} \\
\boldsymbol{v}^{s}
\end{array}\right)^{T} \operatorname{curl}\left(\begin{array}{c}
\boldsymbol{u}^{c} \\
\boldsymbol{u}^{s}
\end{array}\right)+\omega \sigma\left(\begin{array}{c}
\boldsymbol{v}^{c} \\
\boldsymbol{v}^{s}
\end{array}\right)^{T}\left(\begin{array}{cc}
0 & 1 \\
-1 & 0
\end{array}\right)\left(\begin{array}{l}
\boldsymbol{u}^{c} \\
\boldsymbol{u}^{s}
\end{array}\right)\right] \mathrm{d} \boldsymbol{x},
$$

for $\boldsymbol{u}, \boldsymbol{v} \in \boldsymbol{V}$, and consider the mixed formulation

$$
\begin{aligned}
& k(\boldsymbol{u}, \boldsymbol{v})+b(\boldsymbol{v}, \boldsymbol{\phi})=\int_{\Omega}\left(\begin{array}{l}
\boldsymbol{v}^{c} \\
\boldsymbol{v}^{s}
\end{array}\right)^{T}\left(\begin{array}{l}
\boldsymbol{f}^{c} \\
\boldsymbol{f}^{s}
\end{array}\right) \mathrm{d} \boldsymbol{x}, \quad \forall \boldsymbol{v} \in \boldsymbol{V}, \\
& b(\boldsymbol{u}, \boldsymbol{\psi}) \quad=0, \quad \forall \boldsymbol{\psi} \in \boldsymbol{W} .
\end{aligned}
$$

For the following, we denote the right hand side of the first equation, $\int \boldsymbol{v}^{T} \boldsymbol{f}$, by $\langle F, \boldsymbol{v}\rangle$.

By Remark 26 we know that, if the pair $(\boldsymbol{u}, \boldsymbol{\phi}) \in \boldsymbol{V} \times \boldsymbol{W}$ satisfies (63), $\boldsymbol{u}$ is a solution of equation (57) with test functions $\boldsymbol{v} \in \boldsymbol{V}_{b}$. However, since the source current is divergence-free, i.e. $(\boldsymbol{f}, \boldsymbol{\psi})_{\boldsymbol{L}_{2}}=0, \forall \boldsymbol{\psi} \in \boldsymbol{W}, \boldsymbol{u}$ also satisfies equation (57) for gradient fields as test functions, as can easily be seen.

On the other hand, if we have a divergence-free solution $\boldsymbol{u}$ of (57), the pair $(\boldsymbol{u}, 0)$ solves problem (63). In this sense, the mixed problem (63) and the equation (57) (in the space of solenoidal functions $\boldsymbol{V}_{b}$ ) are equivalent.

Since $\boldsymbol{V}_{b^{b}}$-ellipticity of $k(\cdot, \cdot)$ follows from the norm equivalence $\int \mid$ curl $\left.\cdot\right|^{2} \simeq\|\cdot\|_{\boldsymbol{H}(\text { curl })}^{2}$ in the space of divergence-free functions $\boldsymbol{V}_{b}$ (cf. Lemma 2 and Remark 3), and because the inf-sup-condition can easily be verified, we have the following lemma:

Lemma 28. The mixed problem (63) has a unique solution $(\boldsymbol{u}, \boldsymbol{\phi}) \in \boldsymbol{V} \times \boldsymbol{W}$, where moreover, we have $\boldsymbol{u} \in \boldsymbol{V}_{b}$, i.e. $\boldsymbol{u}$ is divergence-free, and $\boldsymbol{\phi}=0$.

Proof. Unique solvability follows immediately by Theorem 27 , and the additional property $\boldsymbol{u} \in \boldsymbol{V}_{b}, \boldsymbol{\phi}=0$ is easy to see.

By the discussion on equivalence between the mixed problem (63) and the variational equation (57), we have the following corollary:

Corollary 29. Problem (57) is uniquely solvable in the space of weakly divergencefree functions $\boldsymbol{V}_{b}$, even with general test functions $\boldsymbol{v} \in \boldsymbol{V}=\boldsymbol{H}_{0}(\mathbf{c u r l})^{2}$.

\subsection{The Perturbed Problem}

As mentioned previously, we introduce a regularization parameter $\epsilon>0$, what guarantees unique solvability of the perturbed problem in the whole space $\boldsymbol{H}_{0}(\mathrm{curl})$.

In order to prove this statement, we define the bilinear form

$$
k_{\epsilon}(\boldsymbol{u}, \boldsymbol{v}):=\int_{\Omega}\left[\nu \operatorname{curl}\left(\begin{array}{l}
\boldsymbol{v}^{c} \\
\boldsymbol{v}^{s}
\end{array}\right)^{T} \operatorname{curl}\left(\begin{array}{l}
\boldsymbol{u}^{c} \\
\boldsymbol{u}^{s}
\end{array}\right)+\omega \sigma_{\epsilon}\left(\begin{array}{l}
\boldsymbol{v}^{c} \\
\boldsymbol{v}^{s}
\end{array}\right)^{T}\left(\begin{array}{cc}
0 & 1 \\
-1 & 0
\end{array}\right)\left(\begin{array}{l}
\boldsymbol{u}^{c} \\
\boldsymbol{u}^{s}
\end{array}\right)\right] \mathrm{d} \boldsymbol{x},
$$


with $\sigma_{\epsilon}(\boldsymbol{x})=\max \{\sigma(\boldsymbol{x}), \epsilon\}(56)$. The perturbed problem

$$
\begin{aligned}
& \text { Find }\left(\boldsymbol{u}_{\epsilon}, \phi_{\epsilon}\right) \in \boldsymbol{V} \times \boldsymbol{W} \text { : } \\
& k_{\epsilon}\left(\boldsymbol{u}_{\epsilon}, \boldsymbol{v}\right)+b\left(\boldsymbol{v}, \boldsymbol{\phi}_{\epsilon}\right)=\langle F, \boldsymbol{v}\rangle, \quad \forall \boldsymbol{v} \in \boldsymbol{V}, \\
& b\left(\boldsymbol{u}_{\epsilon}, \boldsymbol{\psi}\right) \quad=0, \quad \forall \boldsymbol{\psi} \in \boldsymbol{W},
\end{aligned}
$$

with the linear form $F$ as in (63), satisfies the assumptions of Theorem 27 and thus is well posed in $\boldsymbol{H}_{0}(\mathbf{c u r l})^{2} \times \boldsymbol{W}$, just like the original problem (63).

Hence we immediately conclude unique solvability of the perturbed problem in $\boldsymbol{H}_{0}(\mathbf{c u r l})^{2}$ :

Lemma 30. There exists a unique solution $\boldsymbol{u}_{\epsilon} \in \boldsymbol{V}$ of the variational equation

$$
k_{\epsilon}\left(\boldsymbol{u}_{\epsilon}, \boldsymbol{v}\right)=\langle F, \boldsymbol{v}\rangle, \quad \forall \boldsymbol{v} \in \boldsymbol{V},
$$

and $\boldsymbol{u}_{\epsilon}$ is divergence-free, i.e. $\boldsymbol{u}_{\epsilon} \in \boldsymbol{V}_{b}$.

Proof. Existence of a solution $\boldsymbol{u}_{\epsilon} \in \boldsymbol{V}_{b}$ and uniqueness in $\boldsymbol{V}_{b}$ is guaranteed by the equivalence with the mixed problem. Suppose now that $\tilde{\boldsymbol{u}}=\boldsymbol{u}_{\epsilon}+\boldsymbol{w}$ solves (66) for some $\boldsymbol{w} \in \boldsymbol{W}$. Then we have

$$
\begin{aligned}
0 & =k_{\epsilon}\left(\boldsymbol{u}_{\epsilon}+\boldsymbol{w}, \boldsymbol{v}\right)-\langle F, \boldsymbol{v}\rangle \\
& =k_{\epsilon}\left(\boldsymbol{u}_{\epsilon}, \boldsymbol{v}\right)+k_{\epsilon}(\boldsymbol{w}, \boldsymbol{v})-\langle F, \boldsymbol{v}\rangle=k_{\epsilon}(\boldsymbol{w}, \boldsymbol{v}), \quad \forall \boldsymbol{v} \in \boldsymbol{V} .
\end{aligned}
$$

For gradient fields $\boldsymbol{w} \in \boldsymbol{W}, k_{\epsilon}(\boldsymbol{w}, \boldsymbol{v})$ reduces to

$$
\int_{\Omega} \omega \sigma_{\epsilon}\left(\boldsymbol{w}^{c} \boldsymbol{v}^{s}-\boldsymbol{w}^{s} \boldsymbol{v}^{c}\right) \mathrm{d} \boldsymbol{x}
$$

Since $\omega \neq 0$ and $\sigma_{\epsilon}(\boldsymbol{x})>0, \forall \boldsymbol{x}$

$$
k_{\epsilon}(\boldsymbol{w}, \boldsymbol{v})=0, \quad \forall \boldsymbol{v} \in \boldsymbol{V}
$$

implies $\boldsymbol{w}=0$. Consequently, $\boldsymbol{u}_{\epsilon}$ is unique in $\boldsymbol{V}=\boldsymbol{H}_{0}(\mathbf{c u r l})^{2}$.

Remains to show that the perturbed problem is close to the original equation and that its solution converges to the original solution for $\epsilon \rightarrow 0$.

For elliptic variational problems, the situation of a perturbed bilinear form is covered by Strang's Lemmata on variational crimes [23]. These can be generalized to mixed problems, what provides an estimate of the error $\left\|\boldsymbol{u}-\boldsymbol{u}_{\epsilon}\right\|$ :

Lemma 31. Suppose that both the problem (58) and the problem

$$
\begin{array}{llll}
a_{\epsilon}\left(u_{\epsilon}, v\right)+b\left(v, \phi_{\epsilon}\right) & =\langle F, v\rangle, & & \forall v \in V, \\
b\left(u_{\epsilon}, \psi\right) & =0, & & \forall \psi \in W,
\end{array}
$$

with a perturbed bilinear form $a_{\epsilon}(\cdot, \cdot)$ satisfy the assumptions of Theorem 27. Then

$$
\left\|u-u_{\epsilon}\right\|_{V} \leq C \inf _{v \in V_{b}}\left(\|u-v\|_{V}+\sup _{w \in V_{b}} \frac{a(v, w)-a_{\epsilon}(v, w)}{\|w\|_{V}}\right),
$$

where $u$ is the solution of (58) and $u_{\epsilon}$ the solution of (67).

The constant $C=\max \left\{\left(1+\frac{\mu}{\alpha_{\epsilon}}\right), \frac{1}{\alpha_{\epsilon}}\right\}$ depends on $\alpha_{\epsilon}$, the $V_{b}$-ellipticity constant of $a_{\epsilon}(\cdot, \cdot)$, and on $\mu$, the constant of boundedness of $a(\cdot, \cdot)$. 
Proof. The proof is a simple adaptation to mixed problems of the ideas presented in [23]. Details can be found in [3].

Remark 32. The constant $C$ in Lemma 31 depends on $\alpha_{\epsilon}$, the $V_{b}$-ellipticity constant of the perturbed bilinear form $a_{\epsilon}(\cdot, \cdot)$. We emphasize that in our case, i.e. with $k_{\epsilon}(\cdot, \cdot)$ as defined in $(64), \alpha_{\epsilon}$ does not depend on $\epsilon$, since we have

$$
\begin{aligned}
k_{\epsilon}(\boldsymbol{v}, \boldsymbol{v}) & =\int_{\Omega}\left[\nu|\operatorname{curl} \boldsymbol{v}|^{2}+\omega \sigma_{\epsilon}\left(\boldsymbol{v}^{c} \boldsymbol{v}^{s}-\boldsymbol{v}^{s} \boldsymbol{v}^{c}\right)\right] \mathrm{d} \boldsymbol{x} \\
& =\int_{\Omega} \nu|\operatorname{curl} \boldsymbol{v}|^{2} \mathrm{~d} \boldsymbol{x} \geq c\|\boldsymbol{v}\|_{\boldsymbol{V}}^{2}, \quad \forall \boldsymbol{v} \in \boldsymbol{V}_{b} .
\end{aligned}
$$

Indeed, in the space of divergence-free functions $\boldsymbol{V}_{b}$ we have equivalence between the seminorm $\|\mathbf{c u r l} \boldsymbol{v}\|_{\boldsymbol{L}_{2}}$ and the full norm $\|\boldsymbol{v}\|_{\boldsymbol{V}}=\left(\|\boldsymbol{v}\|_{\boldsymbol{L}_{2}}^{2}+\|\operatorname{curl} \boldsymbol{v}\|_{\boldsymbol{L}_{2}}^{2}\right)^{\frac{1}{2}}$ according to Lemma 2 and Remark 3. Since $\nu$ is assumed to be bounded from below, i.e. $\nu(\boldsymbol{x}) \geq \underline{\nu}>0$ almost everywhere, the estimate (68) holds.

As an immediate consequence, we have an estimate for the error introduced by the regularization:

Lemma 33. The solution of the perturbed variational problem

$$
\text { Find } \boldsymbol{u}_{\epsilon} \in \boldsymbol{V}: \quad k_{\epsilon}\left(\boldsymbol{u}_{\epsilon}, \boldsymbol{v}\right)=\langle F, \boldsymbol{v}\rangle, \quad \forall \boldsymbol{v} \in \boldsymbol{V}=\boldsymbol{H}_{0}(\mathbf{c u r l})^{2}
$$

converges to the solution $\boldsymbol{u}$ of (57) for $\epsilon \rightarrow 0$. More precisely we have

$$
\left\|\boldsymbol{u}-\boldsymbol{u}_{\epsilon}\right\|_{\boldsymbol{V}} \leq \epsilon C\|\boldsymbol{u}\|_{\boldsymbol{L}_{2}}
$$

for some constant $C$ independent of $\epsilon$.

Proof. The proof is simple, since the difference between perturbed and original bilinear form can be estimated in the following way:

$$
\begin{aligned}
\left|k(\boldsymbol{v}, \boldsymbol{w})-k_{\epsilon}(\boldsymbol{v}, \boldsymbol{w})\right| & =\left|\int_{\Omega} \omega\left(\sigma-\sigma_{\epsilon}\right)\left(\begin{array}{c}
\boldsymbol{v}^{c} \\
\boldsymbol{v}^{s}
\end{array}\right)^{T}\left(\begin{array}{cc}
0 & 1 \\
-1 & 0
\end{array}\right)\left(\begin{array}{l}
\boldsymbol{w}^{c} \\
\boldsymbol{w}^{s}
\end{array}\right) \mathrm{d} \boldsymbol{x}\right| \\
& \leq \epsilon \omega\|\boldsymbol{v}\|_{\boldsymbol{L}_{2}}\|\boldsymbol{w}\|_{\boldsymbol{L}_{2}} .
\end{aligned}
$$

With this estimate and the knowledge that $\boldsymbol{u} \in \boldsymbol{V}_{b}$ (cf. Lemma 28), we have

$$
\begin{aligned}
\left\|\boldsymbol{u}-\boldsymbol{u}_{\epsilon}\right\|_{\boldsymbol{V}} & \leq C \inf _{\boldsymbol{v} \in \boldsymbol{V}_{b}}\left(\|\boldsymbol{u}-\boldsymbol{v}\|_{\boldsymbol{V}}+\sup _{\boldsymbol{w} \in \boldsymbol{V}_{b}} \frac{\epsilon \omega\|\boldsymbol{v}\|_{\boldsymbol{L}_{2}}\|\boldsymbol{w}\|_{\boldsymbol{L}_{2}}}{\|\boldsymbol{w}\| \boldsymbol{V}}\right) \\
& \leq \epsilon \omega C\|\boldsymbol{u}\|_{\boldsymbol{L}_{2}},
\end{aligned}
$$

according to Lemma 31.

The results of this section, especially the Lemmata 30 and 33, motivate and justify our procedure of solving a perturbed problem with conductivity $\sigma_{\epsilon}$ instead of $\sigma$. 


\subsection{An Estimate of the Discretization Error}

Finite element discretization of the perturbed variational problem (66) yields an approximation $\boldsymbol{u}_{\epsilon, h}$ of $\boldsymbol{u}_{\epsilon}$. For a complete analysis, it remains to estimate the error between the solution of the original problem $\boldsymbol{u}$ and the finite element approximation for the perturbed problem.

For this task, we require some well-known results:

First we need Cea's Lemma (e.g. [5]), which allows to estimate the discretization error $\left\|\boldsymbol{u}_{\epsilon}-\boldsymbol{u}_{\epsilon, h}\right\|$ by the best approximation error and thus by the interpolation error:

$$
\left\|\boldsymbol{u}_{\epsilon}-\boldsymbol{u}_{\epsilon, h}\right\|_{\boldsymbol{V}} \leq c \inf _{\boldsymbol{v}_{h} \in \boldsymbol{V}_{h}}\left\|\boldsymbol{u}_{\epsilon}-\boldsymbol{v}_{h}\right\|_{\boldsymbol{V}} \leq c\left\|\boldsymbol{u}_{\epsilon}-\Pi_{h} \boldsymbol{u}_{\epsilon}\right\|_{\boldsymbol{V}}
$$

Here, by $\Pi_{h}$ we mean an interpolation operator mapping from $\boldsymbol{H}$ (curl) into the Nédélec finite element space. We point out that the constant $c$ in (71) is independent of $\epsilon$, since $\epsilon$ does not appear in the $\boldsymbol{V}_{b}$-ellipticity constant (cf. Remark 32), nor does the constant of boundedness depend on $\epsilon$. The second tool required is a Clément-type quasi interpolation operator according to [22], which is stable in $\boldsymbol{H}$ (curl):

$$
\left\|\Pi_{h} \boldsymbol{w}\right\|_{H(\text { curl })} \leq c\|\boldsymbol{w}\|_{H(\operatorname{curl})} .
$$

The stability in $\boldsymbol{H}$ (curl) follows directly from the $L_{2}$-stability and the commuting diagram property shown in [22]. This quasi interpolation operator also satisfies the following approximation property for $\boldsymbol{w} \in \boldsymbol{H}^{1}$ with $\operatorname{curl} \boldsymbol{w} \in \boldsymbol{H}^{1}$ $[22]$ :

$$
\left\|\left(I-\Pi_{h}\right) \boldsymbol{w}\right\|_{\boldsymbol{H}(\operatorname{curl})} \leq \operatorname{ch}\left(\|\boldsymbol{w}\|_{\boldsymbol{H}^{1}}^{2}+\|\operatorname{curl} \boldsymbol{w}\|_{\boldsymbol{H}^{1}}^{2}\right)^{\frac{1}{2}} .
$$

These results permit to prove the following estimate:

Lemma 34. Let $\boldsymbol{u} \in \boldsymbol{V}_{b}$ be the solution of problem (57) and $\boldsymbol{u}_{\epsilon, h}$ the solution of the discretized perturbed problem (69). Assume moreover $\boldsymbol{u} \in \boldsymbol{H}^{1}$ and curl $\boldsymbol{u} \in$ $\boldsymbol{H}^{1}$. Then we have

$$
\left\|\boldsymbol{u}-\boldsymbol{u}_{\epsilon, h}\right\|_{\boldsymbol{V}} \leq(\epsilon+h) \cdot c(\boldsymbol{u}),
$$

where $c(\boldsymbol{u})$ is some constant depending on $\boldsymbol{u}$.

Proof. With Lemma 33 and the tools quoted above, the estimate follows easily:

$$
\begin{aligned}
\left\|\boldsymbol{u}-\boldsymbol{u}_{\epsilon, h}\right\|_{\boldsymbol{V}} & \leq\left\|\boldsymbol{u}-\boldsymbol{u}_{\epsilon}\right\|_{\boldsymbol{V}}+\underbrace{\left\|\boldsymbol{u}_{\epsilon}-\boldsymbol{u}_{\epsilon, h}\right\|_{\boldsymbol{V}}}_{\leq c\left\|\left(I-\Pi_{h}\right) \boldsymbol{u}_{\epsilon}\right\|} \\
& \leq \epsilon c\|\boldsymbol{u}\|_{\boldsymbol{L}_{2}}+c\left\|\left(I-\Pi_{h}\right)\left[\left(\boldsymbol{u}_{\epsilon}-\boldsymbol{u}\right)+\boldsymbol{u}\right]\right\|_{\boldsymbol{V}} .
\end{aligned}
$$

The second term can be estimated further by taking advantage of stability (72) and approximation (73) of the quasi interpolation operator:

$$
\begin{aligned}
& \leq \epsilon c\|\boldsymbol{u}\|_{\boldsymbol{L}_{2}}+c\left\|\boldsymbol{u}_{\epsilon}-\boldsymbol{u}\right\|_{\boldsymbol{V}}+c h\left(\|\boldsymbol{u}\|_{\boldsymbol{H}^{1}}^{2}+\|\operatorname{curl} \boldsymbol{u}\|_{\boldsymbol{H}^{1}}^{2}\right)^{\frac{1}{2}} \\
& \leq \epsilon c(\boldsymbol{u})+h c(\boldsymbol{u}) \leq(\epsilon+h) \cdot c(\boldsymbol{u}) .
\end{aligned}
$$


We remark that in general full regularity of the solution $\boldsymbol{u}$ cannot be guaranteed. With the whole domain consisting of several subdomains with different parameters $\nu$ and $\sigma$, the solution can at most be piecewise smooth. As long as the interfaces and outer boundary are smooth, $\boldsymbol{f} \in \boldsymbol{L}_{2}$ leads to $\boldsymbol{u} \in \boldsymbol{H}^{2}\left(\Omega_{j}\right)$ for each subdomain $\Omega_{j}$ (cf. e.g. [10]).

However, corners and edges in the interfaces as well as on the outer boundary lead to singularities (cf. $[8,10])$. In that case, one has to be content with interior regularity in each subdomain together with an analysis of the singularities that can be found for example in the works of Costabel, Dauge and Nicaise, e.g. $[8,9,10]$.

It is worth mentioning that $\boldsymbol{u} \in \boldsymbol{H}^{s}$ with $\operatorname{curl} \boldsymbol{u} \in \boldsymbol{H}^{s}$ for some $s \in(0,1)$ still leads to a convergence estimate of the form

$$
\left\|\boldsymbol{u}-\boldsymbol{u}_{\epsilon, h}\right\|_{\boldsymbol{V}} \leq\left(\epsilon+h^{s}\right) \cdot c(\boldsymbol{u}) .
$$

\section{A Complete Error Estimate}

Finally, we are able to give a complete estimate of the error depending on the number of harmonics in the truncated Fourier series $N$, on the regularization parameter $\epsilon$ and on the discretization parameter $h$.

First, however, we state that the Strang-Lemma can immediately be carried over to the nonlinear problem in the space $\mathbb{V}$ :

Lemma 35. Suppose we have

$$
\begin{array}{ll}
\sup _{\boldsymbol{w} \in \mathbb{V},\|\boldsymbol{w}\|=1}\left\langle K_{\epsilon}(\boldsymbol{u})-K_{\epsilon}(\boldsymbol{v}), \boldsymbol{w}\right\rangle \geq \beta_{\epsilon}\|\boldsymbol{u}-\boldsymbol{v}\|_{\mathbb{V}}, & \forall \boldsymbol{u}, \boldsymbol{v} \in \mathbb{V}, \\
\sup _{\boldsymbol{w} \in \mathbb{V},\|\boldsymbol{w}\|=1}\langle K(\boldsymbol{u})-K(\boldsymbol{v}), \boldsymbol{w}\rangle \leq \gamma\|\boldsymbol{u}-\boldsymbol{v}\|_{\mathbb{V}}, & \forall \boldsymbol{u}, \boldsymbol{v} \in \mathbb{V},
\end{array}
$$

then the difference between the solutions $\boldsymbol{u}$ and $\boldsymbol{u}_{\epsilon}$ of

$$
\begin{aligned}
\langle K(\boldsymbol{u}), \boldsymbol{v}\rangle & =\langle F, \boldsymbol{v}\rangle, & & \forall \boldsymbol{v} \in \mathbb{V}, \\
\left\langle K_{\epsilon}\left(\boldsymbol{u}_{\epsilon}\right), \boldsymbol{v}\right\rangle & =\langle F, \boldsymbol{v}\rangle, & & \forall \boldsymbol{v} \in \mathbb{V},
\end{aligned}
$$

can be estimated by

$$
\left\|\boldsymbol{u}-\boldsymbol{u}_{\epsilon}\right\| \leq C\left(\beta_{\epsilon}, \gamma\right) \inf _{\boldsymbol{w} \in \mathbb{V}}\left(\|\boldsymbol{u}-\boldsymbol{w}\|_{\mathbb{V}}+\sup _{\boldsymbol{v} \in \mathbb{V}} \frac{\left\langle K_{\epsilon}(\boldsymbol{w})-K(\boldsymbol{w}), \boldsymbol{v}\right\rangle}{\|\boldsymbol{v}\|_{\mathbb{V}}}\right) .
$$

Proof. As with Lemma 31, the proof is a simple adaptation to nonlinear problems of the ideas presented in [23]. The linear version can be found in [21].

Remark 36. The prerequisites of Lemma 35 are immediate consequences of the inf-sup- (42) and sup-sup-condition (43). It can easily be checked that the constant $\beta_{\epsilon}$ is actually independent of $\epsilon$, just like in the linear harmonic problem (cf. Remark 32). Consequently, we get convergence for $\epsilon \rightarrow 0$ :

$$
\left\|\boldsymbol{u}-\boldsymbol{u}_{\epsilon}\right\|_{\mathbb{V}} \leq \epsilon C\|\boldsymbol{u}\|_{\mathbb{V}}
$$


Theorem 37. Let the assumptions of Theorem 25 be satisfied, and let us denote by $\boldsymbol{u} \in \mathbb{V}$ the unique periodic steady state solution of the eddy current problem (5), i.e. the solution of (45). Let $\boldsymbol{u}_{N} \in \mathbb{V}_{N} \subset \mathbb{V}$ be the solution of the multiharmonic problem with $N$ harmonics (52), and denote by $\boldsymbol{u}_{N, \epsilon, h} \in\left(\boldsymbol{V}_{h}^{2}\right)^{N} \subset \mathbb{V}_{N}$ its approximation, which solves the discretized perturbed problem. Suppose that $\boldsymbol{u}$ is smooth: $\boldsymbol{u}_{k}^{c / s} \in \boldsymbol{H}^{1}$ with $\operatorname{curl} \boldsymbol{u}_{k}^{c / s} \in \boldsymbol{H}^{1}$, for all $k$.

Then we have the following estimate:

$$
\left\|\boldsymbol{u}-\boldsymbol{u}_{N, \epsilon, h}\right\|_{\mathbb{V}} \leq\left(\epsilon+h+N^{-1}\right) c(\boldsymbol{u}),
$$

where $c(\boldsymbol{u})$ is a constant depending on the exact solution $\boldsymbol{u}$, and on $\boldsymbol{\omega}$ as well.

Proof. The proof combines the previous results on Fourier series convergence and on the discretization error. It rests upon the observation that the estimates in $\epsilon$ and $h$ can be directly carried over to the nonlinear problem in the spaces $\mathbb{V}$ and $\mathbb{V}_{N}$, respectively. This is possible, since we can generalize the StrangLemma to the space $\mathbb{V}$ (Lemma 35).

As a consequence, the error between $\boldsymbol{u}$ and the discrete approximation $\boldsymbol{u}_{N, \epsilon, h}$ can be estimated by the triangle inequality:

$$
\begin{aligned}
\left\|\boldsymbol{u}-\boldsymbol{u}_{N, \epsilon, h}\right\|_{\mathbb{V}} & \leq\left\|\boldsymbol{u}-\boldsymbol{u}_{N}\right\|_{\mathbb{V}}+\left\|\boldsymbol{u}_{N}-\boldsymbol{u}_{N, \epsilon, h}\right\|_{\mathbb{V}} \\
& \leq c(\boldsymbol{u}) N^{-1}+c\left(\boldsymbol{u}_{N}\right)(\epsilon+h) \\
& \leq\left(\epsilon+h+N^{-1}\right) c(\boldsymbol{u}) .
\end{aligned}
$$

\section{Concluding Remarks}

In this paper we presented a complete analysis of nonlinear eddy current problems consisting of conducting and non-conducting domains. For their numerical treatment, we suggested and analyzed a multiharmonic solution technique that takes advantage of the periodicity of the solution. The errors due to the truncated Fourier series expansion as well as spatial discretization and regularization were rigorously estimated.

Fast solvers for the system defining the discrete Fourier coefficients and other aspects of the numerical simulation as well as numerical results demonstrating the efficiency of the multiharmonic approach in combination with multigrid techniques will be presented in a foregoing paper.

\section{References}

[1] C. Amrouche, C. Bernardi, and M. Dauge, Vector potentials in threedimensional nonsmooth domains, Math. Meth. Appl. Sci. 21 (1998), 823864 . 
[2] I. Babuška, Error bounds for the finite element method, Numer. Math. 16 (1971), 322-333.

[3] F. Bachinger, Multigrid Solvers for 3D Multiharmonic Nonlinear Magnetic Field Computations, Diploma thesis, Johannes Kepler University Linz, 2003.

[4] F. Bachinger, M. Kaltenbacher, and S. Reitzinger, An Efficient Solution Strategy for the HBFE Method, Proceedings of the IGTE '02 Symposium Graz, Austria, 2002, pp. 385-389.

[5] D. Braess, Finite Elements: Theory, Fast Solvers and Applications in Solid Mechanics, 2nd ed., Cambridge University Press, Cambridge, 2001.

[6] F. Brezzi, On the existence, uniqueness and approximation of saddle-point problems arising from Lagrangian multipliers, RAIRO Anal. Numér. 8 (1974), 129-151.

[7] A. Buffa, M. Costabel, and D. Sheen, On traces for $\boldsymbol{H}(\mathbf{c u r l}, \Omega)$ in Lipschitz domains, J. Math. Anal. Appl. 276/2 (2002), 845-876.

[8] M. Costabel, M. Dauge, and S. Nicaise, Singularities of Maxwell interface problems, RAIRO Modél. Math. Anal. Numér. 33 (1999), 627-649.

[9] _ Singularities of eddy current problems, Preprint NI03019, Newton Institute Cambridge, 2003.

[10] _ Corner singularities of Maxwell interface and eddy current problems, Operator Theoretical Methods and Applications to Mathematical Physics - The Erhard Meister Memorial Volume (I. Gohberg, A. F. dos Santos, F.-O. Speck, F.S. Teixeira, and W. Wendland, eds.), Operator Theory: Advances and Applications, Birkhäuser, 2003.

[11] H. W. Engl and E. Lindner, A combined boundary value and transmission problem arising from the calculation of eddy currents: well-posedness and numerical treatment, Journ. Appl. Math. Phys. 35 (1984), 289-307.

[12] L. C. Evans, Partial Differential Equations, Graduate studies in mathematics, vol. 19, American Mathematical Society, Providence, Rhode Island, 1998.

[13] H. de Gersem, H. V. Sande, and K. Hameyer, Strong coupled multiharmonic finite element simulation package, COMPEL 20 (2001), 535-546.

[14] J. Gyselinck, P. Dular, C. Geuzaine, and W. Legros, Harmonic-Balance Finite-Element Modeling of Electromagnetic Devices: A Novel Approach, IEEE Transactions on Magnetics 38 (2002), 521-524.

[15] N. Ida and P. A. Bastos, Electromagnetics and Calculation of Fields, Springer, New York, 1997. 
[16] A. Jack and B. Mecrow, Methods for magnetically nonlinear problems involving significant hysteresis and eddy currents, IEEE Transactions on Magnetics 26 (1990), 424-429.

[17] J. Kacur, Method of Rothe in Evolution Equations, B. G. Teubner, Leipzig, 1985.

[18] G. Paoli, O. Bíro, and G. Buchgraber, Complex representation in nonlinear time harmonic eddy current problems, IEEE Transactions on Magnetics 34 (1998), 2625-2628.

[19] C. Pechstein, Multigrid-Newton-Methods for Nonlinear Magnetostatic Problems, Diploma thesis, Johannes Kepler University Linz, in preparation.

[20] S. Reitzinger, B. Kaltenbacher, and M. Kaltenbacher, A Note on the Approximation of $B-H$ Curves for Nonlinear Magnetic Field Computations, SFB-Report No. 02-30, Johannes Kepler Universiy Linz, SFB "Numerical and Symbolic Scientific Computing", 2002.

[21] J. E. Roberts and J.-M. Thomas, Mixed and Hybrid Methods, Handbook of Numerical Analysis II: Finite Element Methods (Part 1) (P. G. Ciarlet and J. L. Lions, eds.), North-Holland, Amsterdam, 1991.

[22] J. Schöberl, Commuting Quasi-Interpolation Operators for Mixed Finite Elements, Technical Report ISC-01-10-MATH, Texas A\&M University, 2001.

[23] G. Strang, Variational crimes in the finite element method, The Mathematical Foundations of the Finite Element Method with Applications to Partial Differential Equations (A. K. Aziz, ed.), Academic Press, New York, 1972.

[24] L. Vandevelde, J. Gyselinck, and J. Melkebeek, Steady-state finite element analysis in the frequeny domain of squirrel-cage induction motors, Proceedings of the SPEEDAM '94 Symposium, Taormina, Italy, 1994, pp. 29-34.

[25] S. Yamada and K. Bessho, Harmonic field calculation by the combination of finite element analysis and harmonic balance method, IEEE Transactions on Magnetics 24 (1988), 2588-2590.

[26] E. Zeidler, Nonlinear Functional Analysis and its Applications II/A: Linear Monotone Operators, Springer, New York, 1990.

[27] _ Nonlinear Functional Analysis and its Applications II/B: Nonlinear Monotone Operators, Springer, New York, 1990. 